\title{
The Mu2e: The Muon to Electron Conversion Experiment
}

\section{Presented By Sophie Charlotte Middleton \\ From The University of Manchester \\ At the Fermilab Users Meeting}

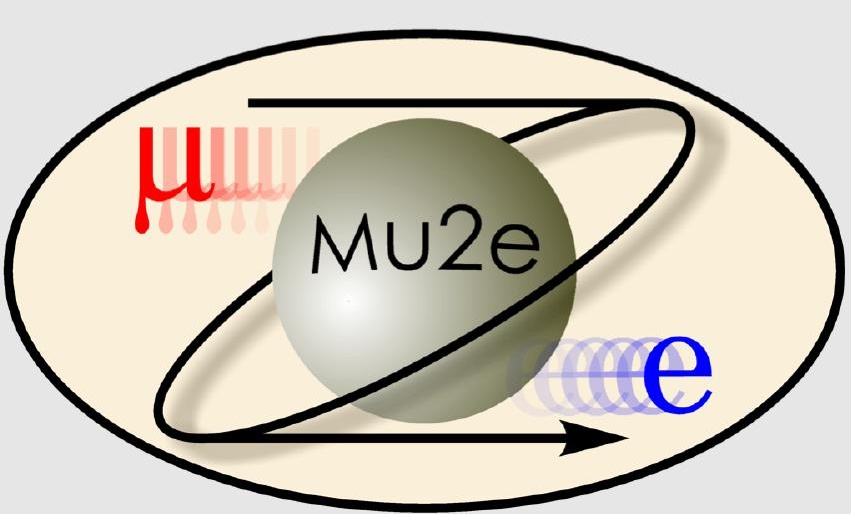

This document was prepared by Mu2e collaboration using the resources of the Fermi National Accelerator Laboratory (Fermilab), a U.S. Department of Energy, Office of Science, HEP User Facility. Fermilab is managed by Fermi Research Alliance, LLC (FRA), acting under $13^{\text {th }}$ June 2019 Contract No. DE-AC02-07CH11359.
MANCHESTER 1824

The University of Manchester 


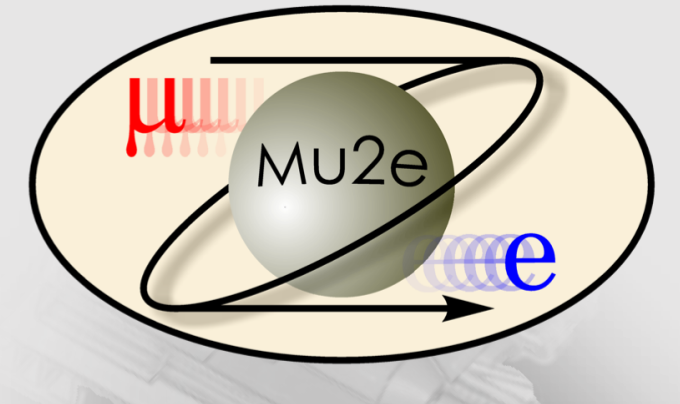

What are we looking for?

Experimental Method Employed by Mu2e.

Hardware and Infrastructure.

Current Status of Hardware.

Looking Further ahead.

Questions? 
To elucidate the mechanism responsible for NP-must look at relative rates of CLFV for many processes. Intense muon beams put muon sector at forefront. to NP effects:

\begin{tabular}{|c|c|c|c|}
\hline Mode & $\begin{array}{c}\text { Current Limit } \\
\text { (at } 90 \% \mathrm{CL})\end{array}$ & Future Limit & $\begin{array}{c}\text { Future } \\
\text { Experiment/s }\end{array}$ \\
\hline $\boldsymbol{\mu}^{ \pm} \rightarrow \boldsymbol{e}^{ \pm} \boldsymbol{\gamma}$ & $5.7 \times 10^{-13[1]}$ & $4 \times 10^{-14}$ & MEG II[4] \\
\hline $\boldsymbol{\mu}^{-} \boldsymbol{N} \rightarrow \boldsymbol{e}^{-} \boldsymbol{N}$ & $7 \times 10^{-13[2]}$ & $10^{-15} / 10^{-17}$ & $\begin{array}{c}\text { COMET }^{[5]} \text { Phase } 1 / \\
\text { Mu2e }\end{array}$ \\
\hline $\boldsymbol{\mu}^{+[6]} \rightarrow \boldsymbol{e}^{+} \boldsymbol{e}^{+} \boldsymbol{e}^{-}$ & $\sim 10^{-12[3]}$ & $10^{-15} \sim 10^{-16}$ & Mu3e $^{[7]}$ \\
\hline
\end{tabular}
3 muon CLFV channels with complementary sensitivity

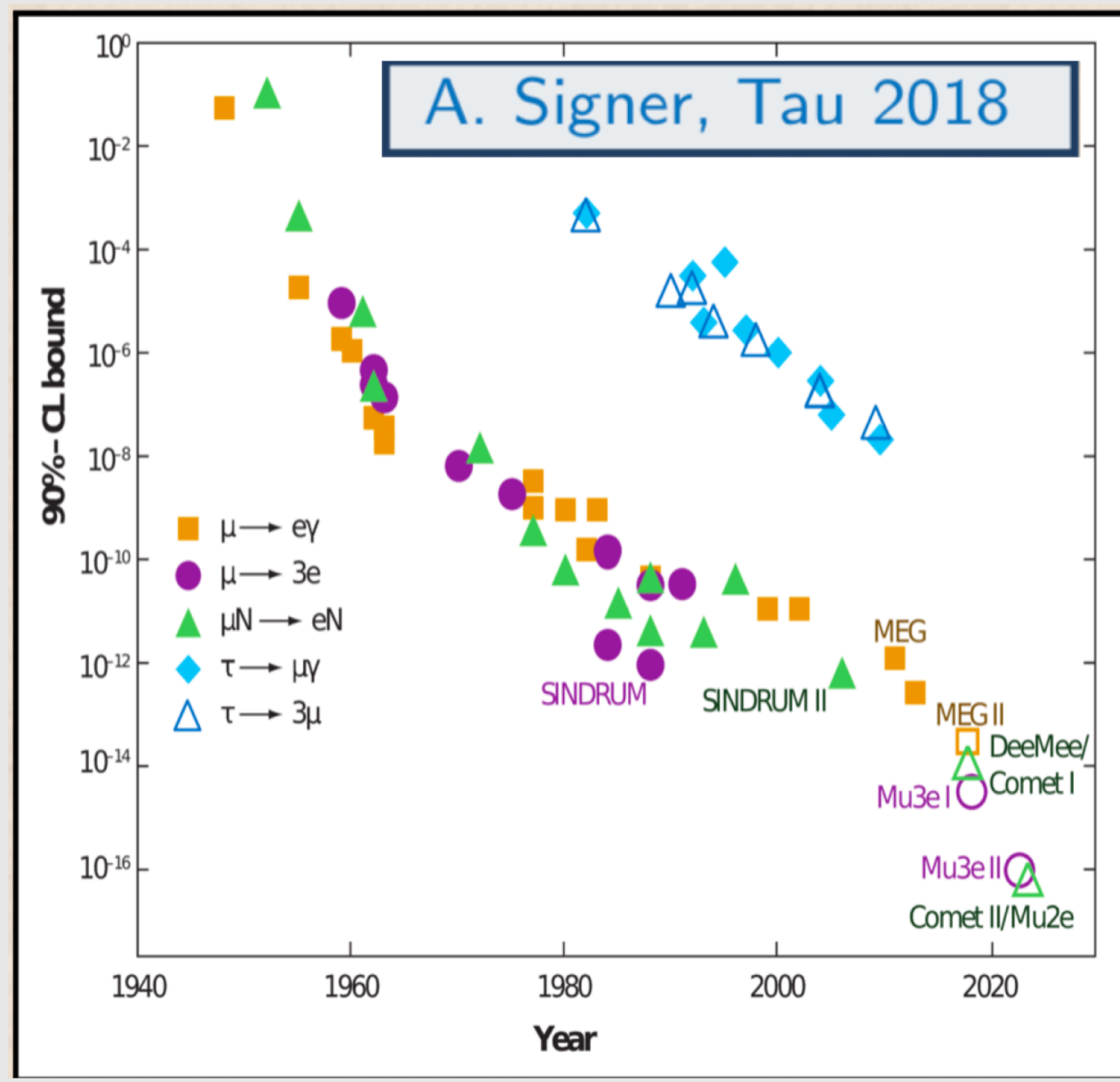

[1] J. Adam et al. (EG Collaboration), Phys. Rev. Lett. 110, 20 (2013).

[2] W. Bertl et al. (SINDRUM-II Collaboration),

Eur. Phys. J. C47, 337 (2006).

[3] U. Bellgardt et al., (SINDRUM

Collaboration), Nucl. Phys. B299, 1 (1988).

[4] A.M. Baldini et al., "MEG Upgrade Proposal", arXiv:1301.7225v2 [physics.ins-

1.det].

[5] Y. Kuno et al., "COMET Proposal" (2007)

[6] Mu2e TDR, arXiv:1501.05241

Proceedings Supplements

Volumes 248-250, March-May

2014, Pages 35-40

[8] Marciano, W. J., T. Mori, and J. M. Roney (2008)

\section{MANCHESTER 1824}

The University of Manchester 
Estimate sensitivity CLFV process in model independent manner by adding 2 different LFV effective operators to the SM Lagrangian:

$$
\begin{aligned}
& \mathcal{L}_{C L F V}=\frac{m_{\mu}}{(1+\kappa) \Lambda^{2}} \bar{\mu}_{R} \sigma_{\mu \nu} e_{L} F^{\mu \nu}+\frac{\kappa}{(1+\kappa) \Lambda^{2}} \bar{\mu}_{L} \gamma_{\mu} e_{L}\left(\sum_{q=u, d} \bar{q}_{L} \gamma_{\mu} q_{L}\right) \\
& \text { "Photonic" } \\
& \mu^{ \pm} \rightarrow e^{ \pm} \gamma, \mu \rightarrow e e e \\
& \mu^{-} N \rightarrow e^{-} N \\
& \Lambda=\text { the effective mass scale of NP, } \\
& K=\text { controls the relative contribution of the two terms } \\
& \text { e.g SUSY } \kappa=0
\end{aligned}
$$

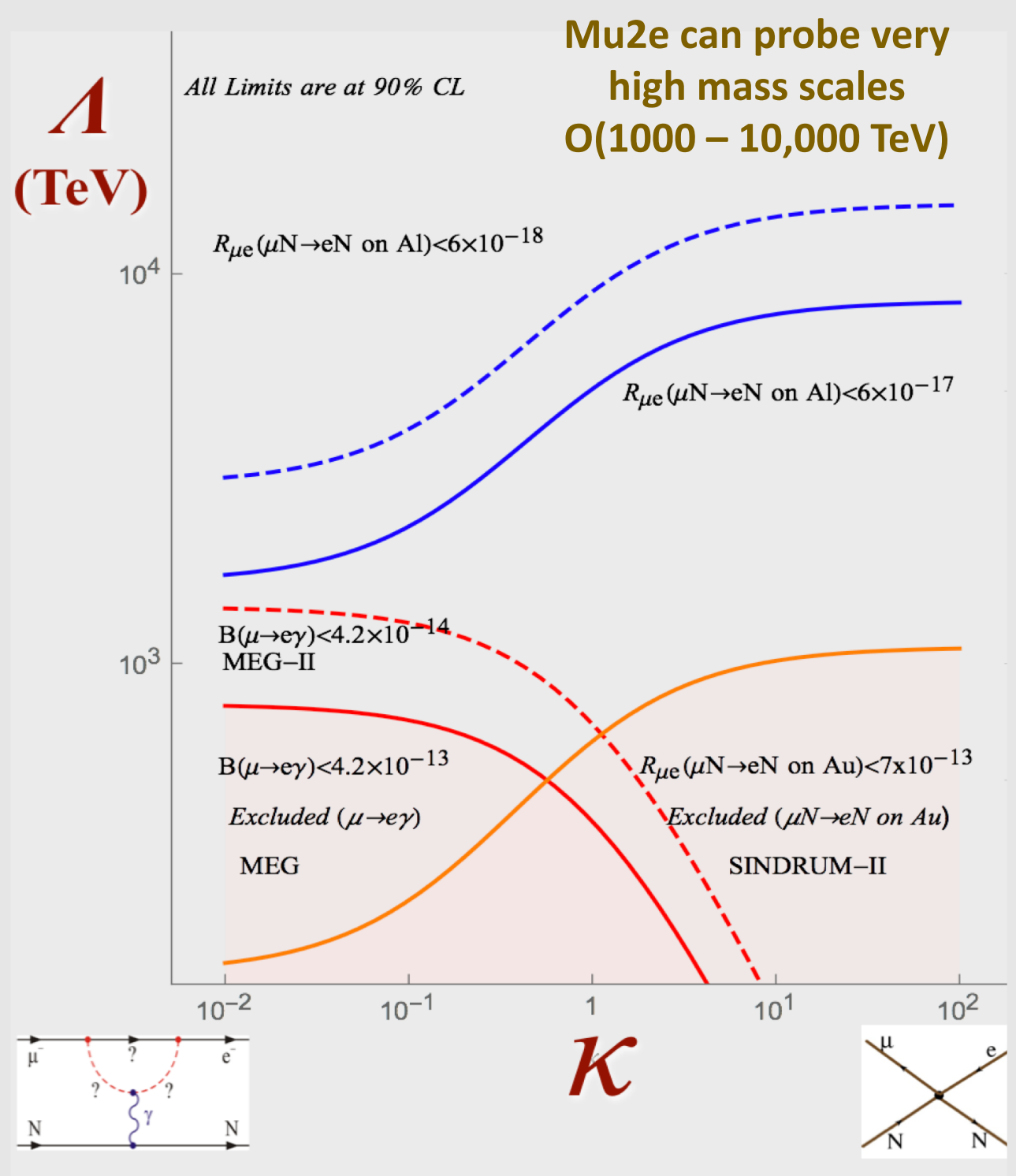

For more detailed theoretical understanding: Eur. Phys. J C75 (2015) no.12, 579 
\& The SINDRUM-II results was limited by 2 main factors:

$\diamond$ Backgrounds from prompt pions,

$\diamond$ The muon stopping rate ( $\sim 10^{7} \mu / \mathrm{s}-$ with a $\sim 1 \mathrm{MW}$ beam).

$\diamond$ Mu2e must address these issues to improve limit on $\boldsymbol{R}_{\mu e}$.

Following the proposal by V. Lobashev \& R. Djilkibaev (Sov. J. Nucl. Phys. 49(2), 384 (1989)), Mu2e will:

\& Utilize a pulsed proton beam \& delayed "gate window" - Eliminates pion induced backgrounds.

Use intense muon source $-10^{10}$ muons/s -3 year run equates to $10^{18}$ stopped muons.

$\diamond$ Use superconducting solenoids - For efficient muon collection and transport to stopping target. 
Intrinsic :

Scale with number of stopped muons.

\section{Late arriving :}

Scale with number of late protons/extinction performance

\begin{tabular}{l|l|l|l}
\hline Type & Source & Mitigation & Yield \\
\hline Intrinsic & $\begin{array}{l}\text { Decay in Orbit } \\
\text { (DIO) }\end{array}$ & Tracker Resolution & $\begin{array}{l}0.144 \pm 0.028 \text { (stat) } \\
\pm 0.11 \text { (sys) }\end{array}$ \\
\hline $\begin{array}{l}\text { Late } \\
\text { Arriving }\end{array}$ & Pion Capture & Beam Structure & $\begin{array}{l}0.021 \text { (stat) } \pm 0.001 \pm \\
0.002 \text { (sys) }\end{array}$ \\
& Pion Decay in & - & $\begin{array}{l}0.001 \text { (stat) } \pm<0.001 \\
\text { (sys) }\end{array}$ \\
& Flight & & $\begin{array}{l}0.04 \pm 0.022 \text { (stat) } \pm \\
0.020 \text { (sys) }\end{array}$ \\
\hline Other & Anti-proton & Thin windows & $0.209 \pm 0.0022$ (stat) \pm \\
& & & 0.055 (sys)
\end{tabular}

MANCHESTER 1824 


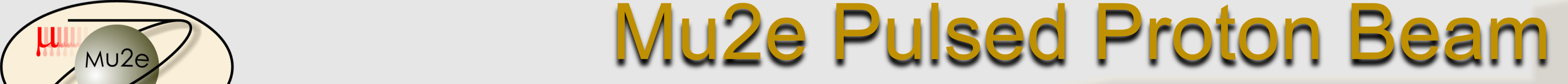

\& Mu2e uses a pulsed beam with a long interval between pulses.

$\diamond$ Search window begins 700ns in-this suppresses prompt backgrounds e.g. pion background reduced by $>10^{-11}$.

$\diamond$ Must eliminate protons that arrive late. These can give rise to additional pion backgrounds in delayed live window.

$\diamond$ AC Dipole in beamline helps ensure $<10^{-10}$. Extinction monitor measures.

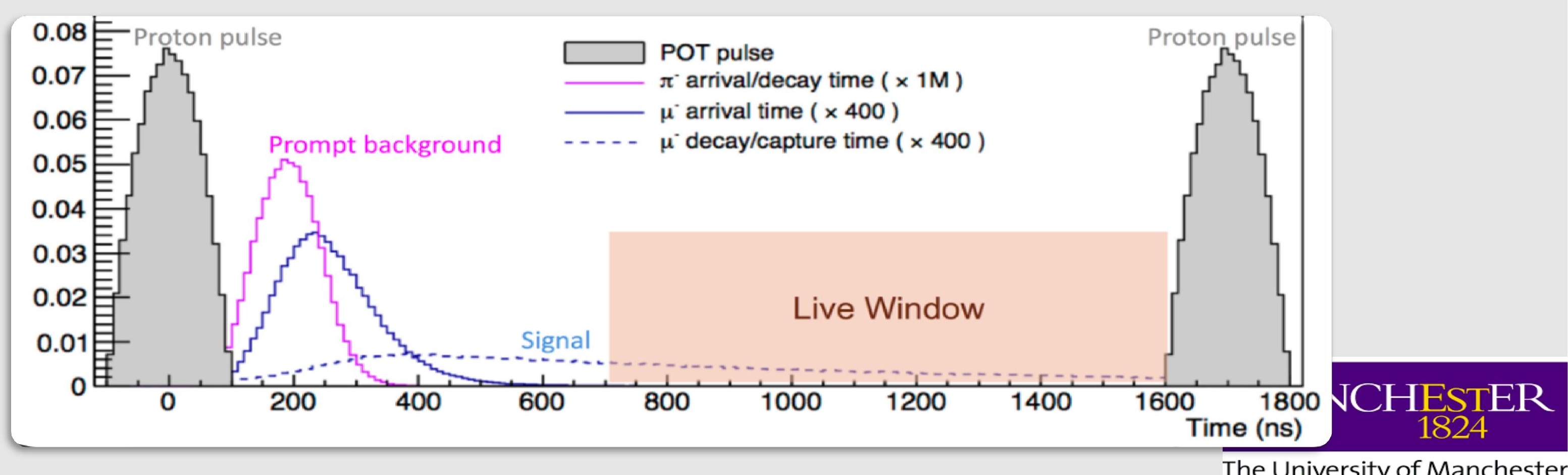




\section{0 The Importance of Tracker Resolution}

$\diamond$ In $39 \%$ of stopped muons will decay in orbit (DIO).

$\diamond$ This is a $\mathbf{3}$ body decay. In free decay maximum electron energy is far below our signal energy (104.97 MeV).

$\diamond$ The free decay spectrum is distorted by the presence of the nucleus.
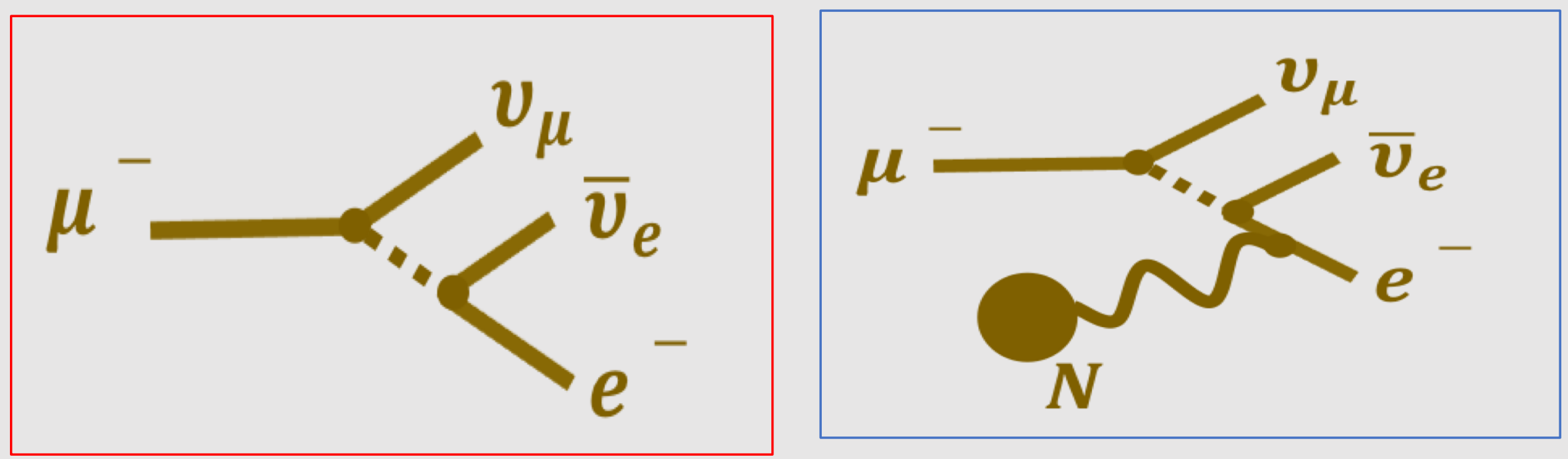


\section{The Importance of Tracker Resolution}

\section{Mu2e requires excellent tracker resolution of $<200 \mathrm{keV} / \mathrm{c}$ to suppress DIO background.}
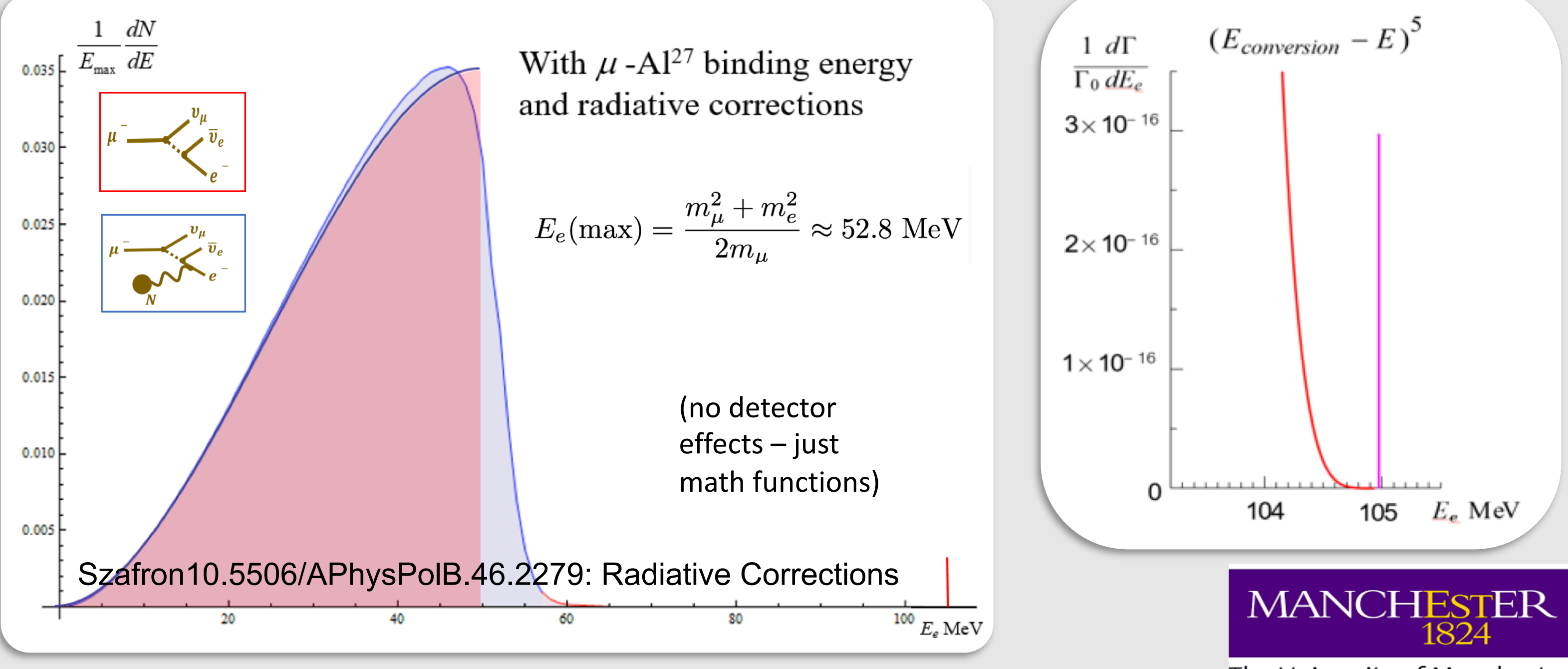


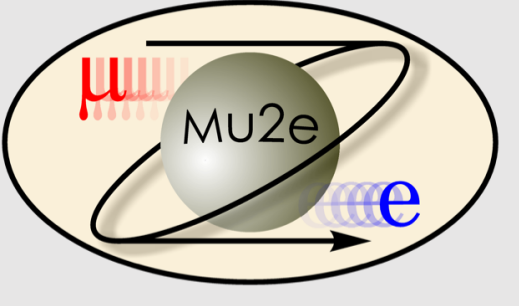

\& In order to reach design sensitivity Mu2e will use:

$\nLeftarrow$ Low mass straw tracker provides excellent momentum resolution $<200 \mathrm{keV} / \mathrm{c}$ on electrons.

Pulsed proton beam with:

$\&$ Narrow proton pulses $(<+/-125 \mathrm{~ns})$.

$\&$ Very few out-of-time protons $\left(<10^{-10}\right)$.

Anti-proton backgrounds reduced by thin anti-proton windows in beamline.

\& Passive and active shielding means high cosmic ray veto efficiency (>99.99\%). 

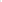
A low mass, annular, highly segmented detector is used to:

1. Minimizes scattering and energy loss :

Entire Detector Solenoid held under vacuum ( $10^{-4}$ torr). Ultra low mass tracker.

2. Minimizes Background Tracker - excludes low momentum electrons via hollow centre:

Inner $38 \mathrm{~cm}$ instrumented .

Reduces need to reject $\sim 10^{18}$ to $\sim 10^{5}$.

Blind to $>99 \%$ of DIO spectrum .

3. Handle high rates and provide high-precision momentum measurements :

Highly segmented design.

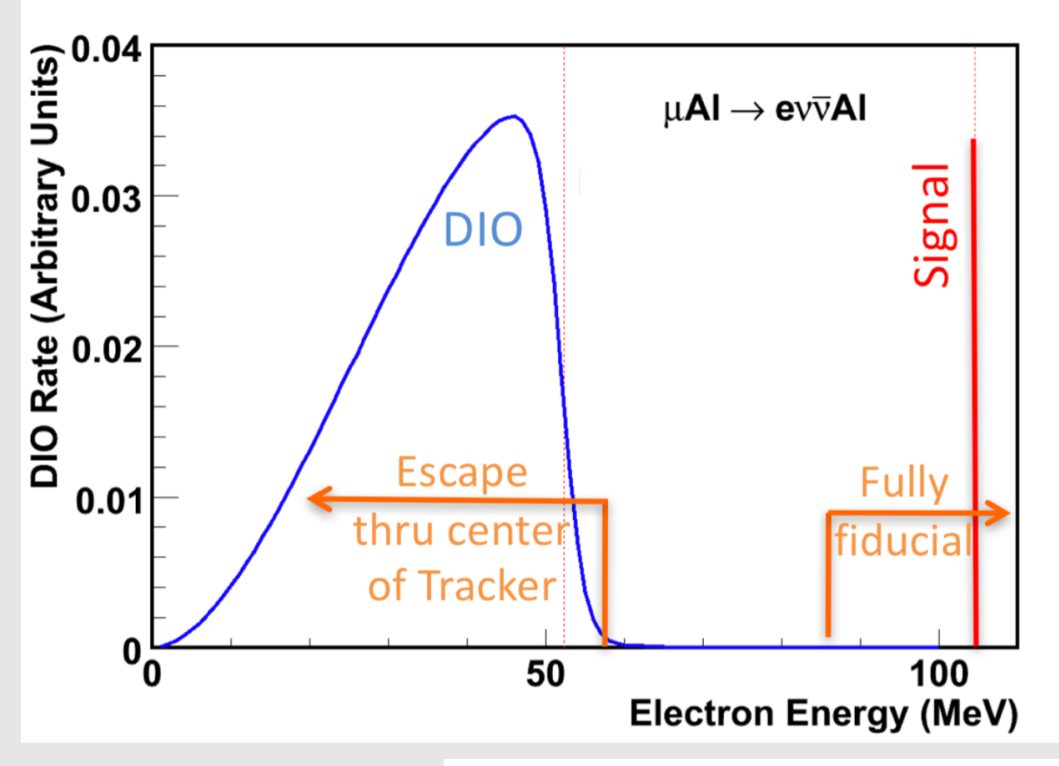

MANCHESTER 

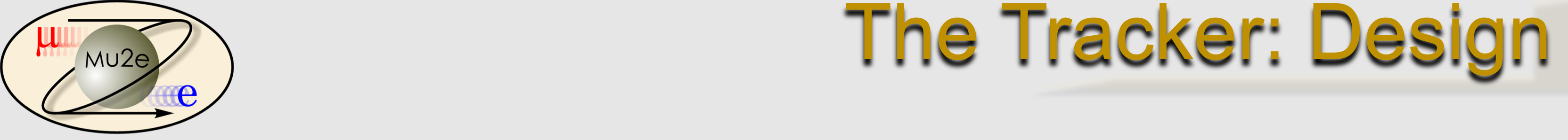

Tracker is constructed from self-supporting panels of low mass straws tubes detectors $\diamond 18$ stations, 2 planes per station, 6 panels per plane, 96 straws per panel.

\& Straw drift tubes aligned transverse to the axis of the Detector Solenoid.

$1 \mathrm{~m}, 5 \mathrm{~mm}$ diameter straw

Walls: $12 \mathrm{~mm}$ Mylar $+3 \mathrm{~mm}$ epoxy

$25 \mathrm{~mm}$ Au-plated $\mathrm{W}$ sense wire

$33-117 \mathrm{~cm}$ in length

$80: 20 \mathrm{Ar}: \mathrm{CO}_{2}$ with $\mathrm{HV}<1500 \mathrm{~V}$
The Straws:

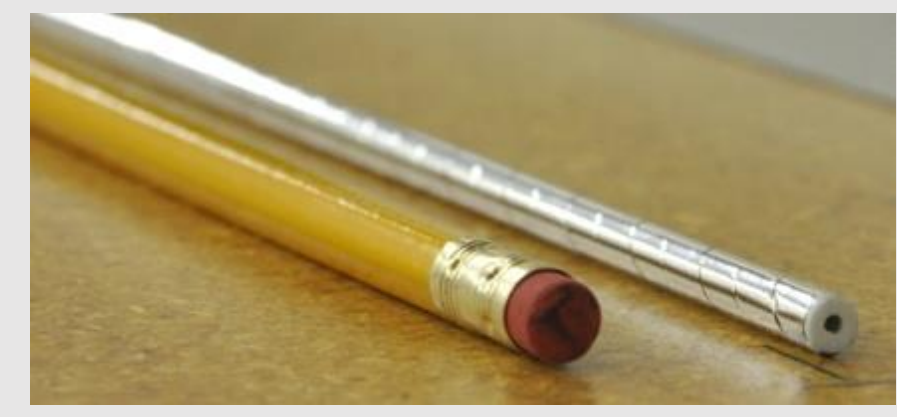

The Tracker:
Panels

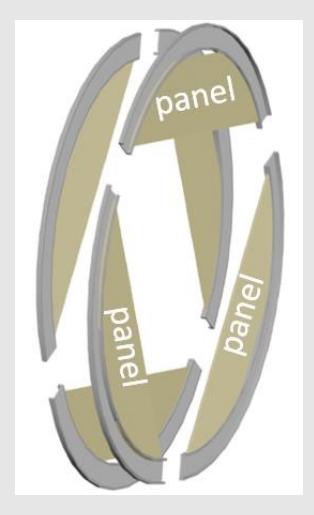

Plane

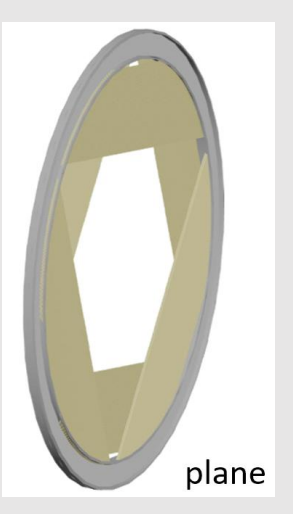

Station

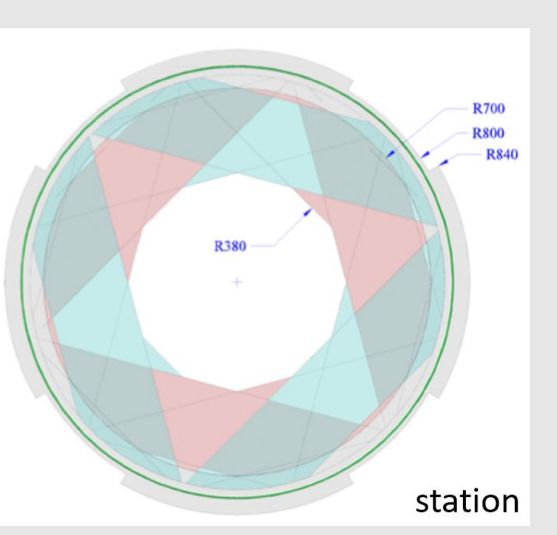

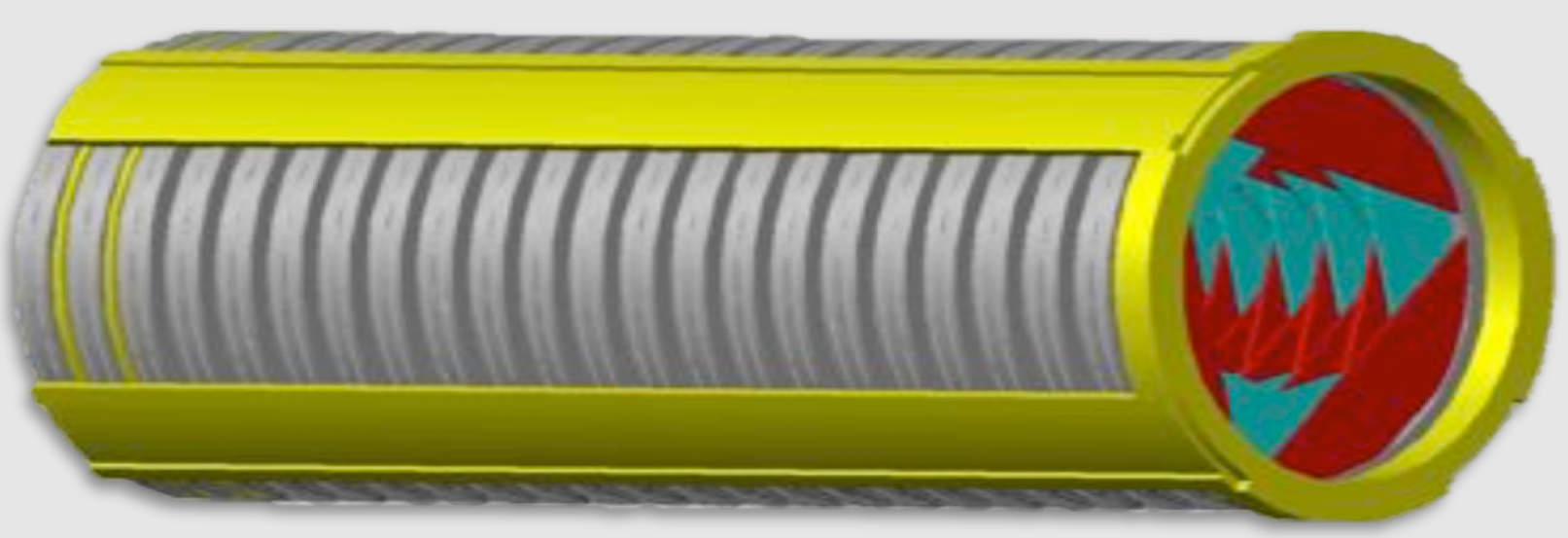

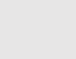



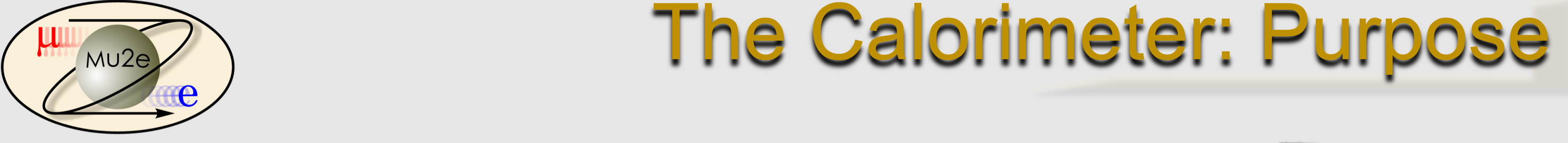

The calorimeter is vital for providing:

\& Particle identification,

\& Fast online trigger filter,

\& Accurate timing information for background rejection

seed for track reconstruction.

The calorimeter must:

\& Have a large acceptance.

\& Provide time resolution $<0.5 \mathrm{~ns}$,

\& Energy resolution $<10 \%$;

$\nLeftarrow$ Position resolution of $1 \mathrm{~cm}$.

\& Function in region with radiation exposure up to 20Gy/crystal/year and with neutron flux $10^{11} / \mathrm{cm}^{2}$

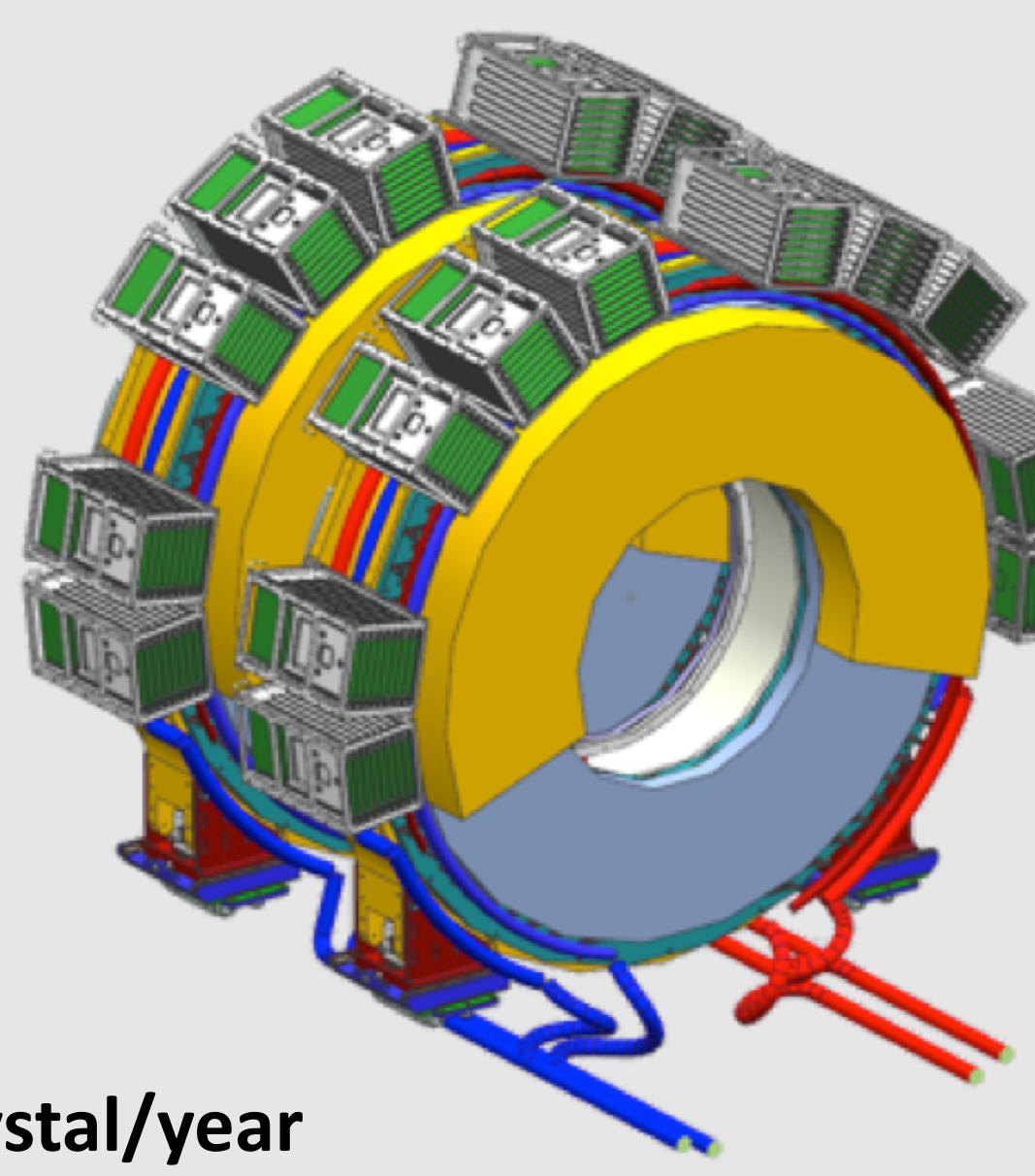




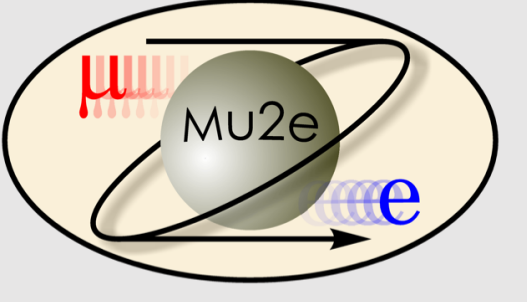

Prototype panels complete, ramping-up production line.

Full-sized pre-production panels assembled and tested.

Multiple panels have been assembled into a prototype station.

Final straw production is currently underway.

FEE Readout QA taking place at LBNL/Berkeley, this summer.

University of Minnesota

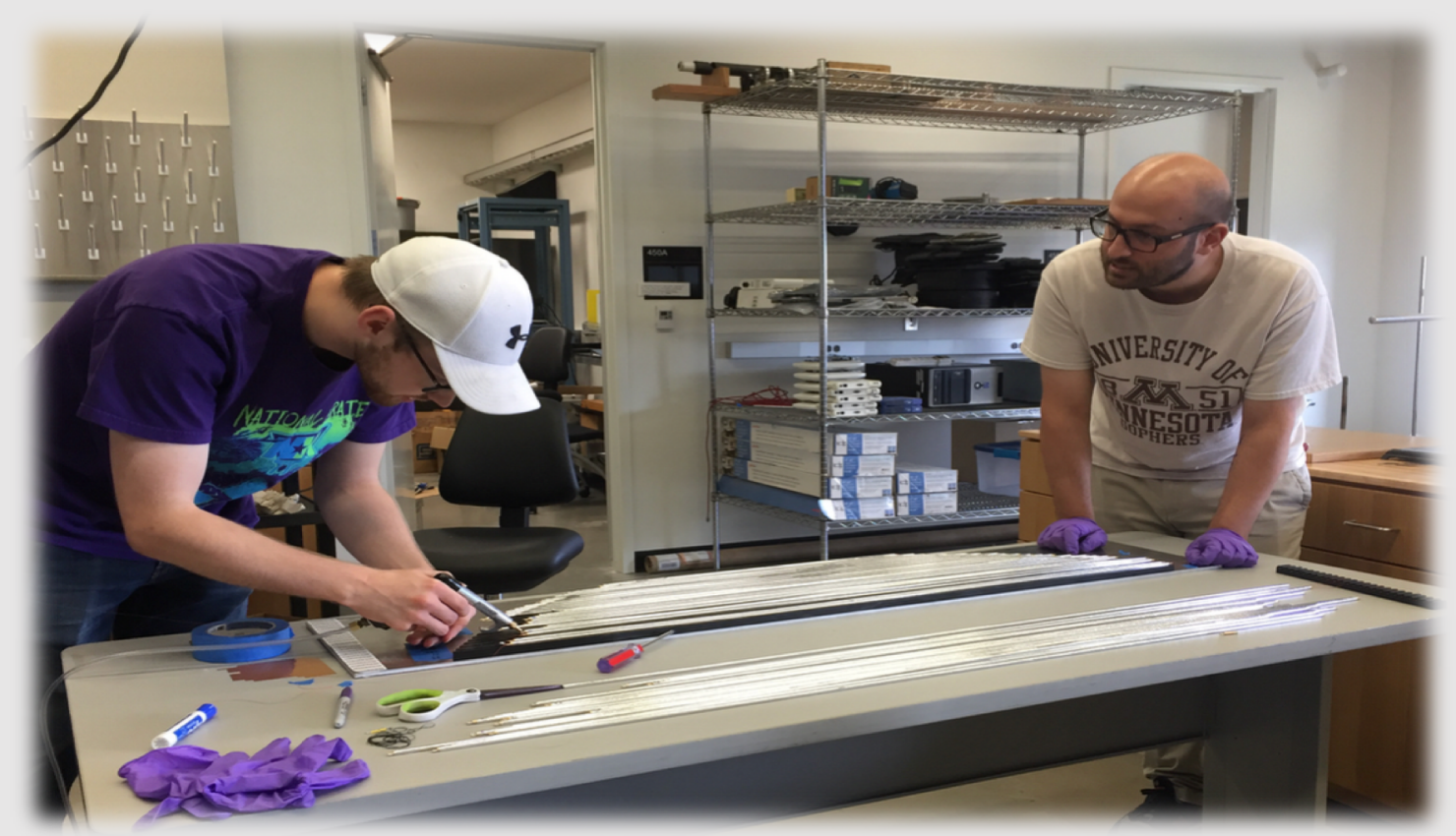

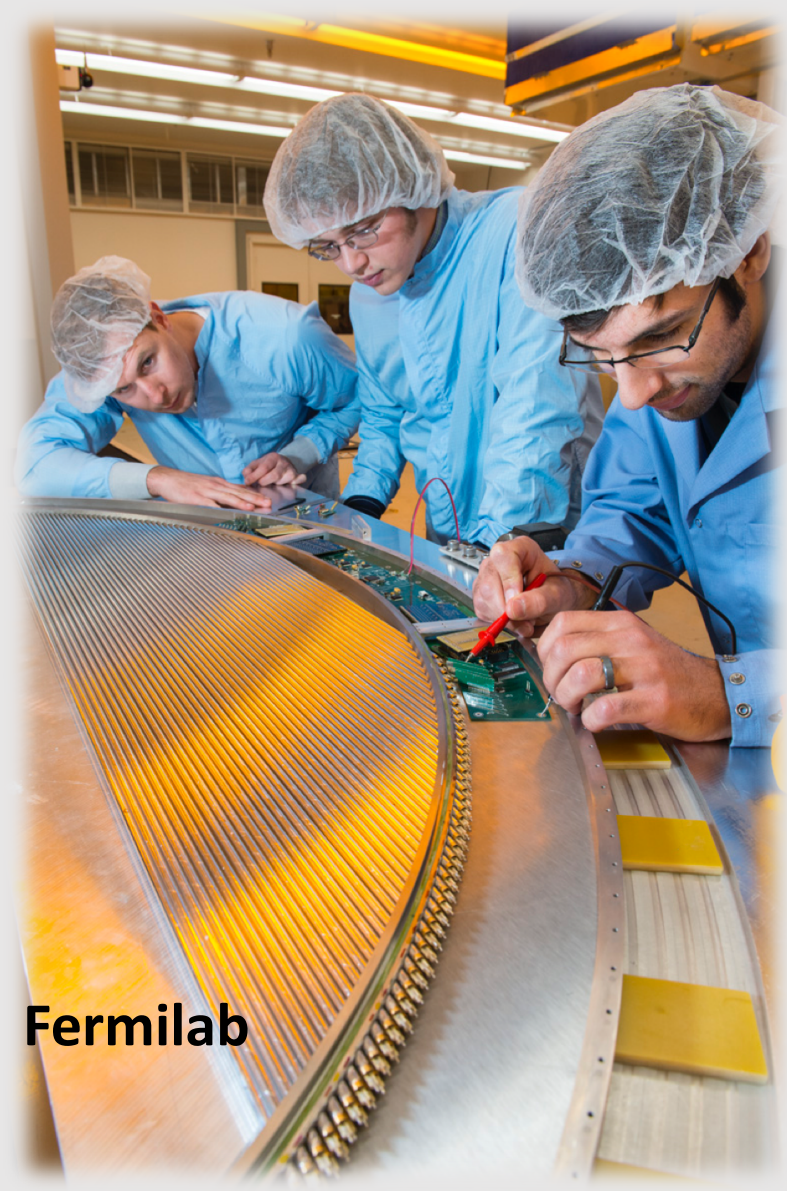

MANCHESTER 1824 


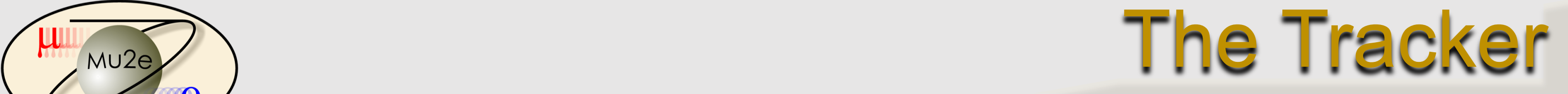

\section{8 channel prototype}

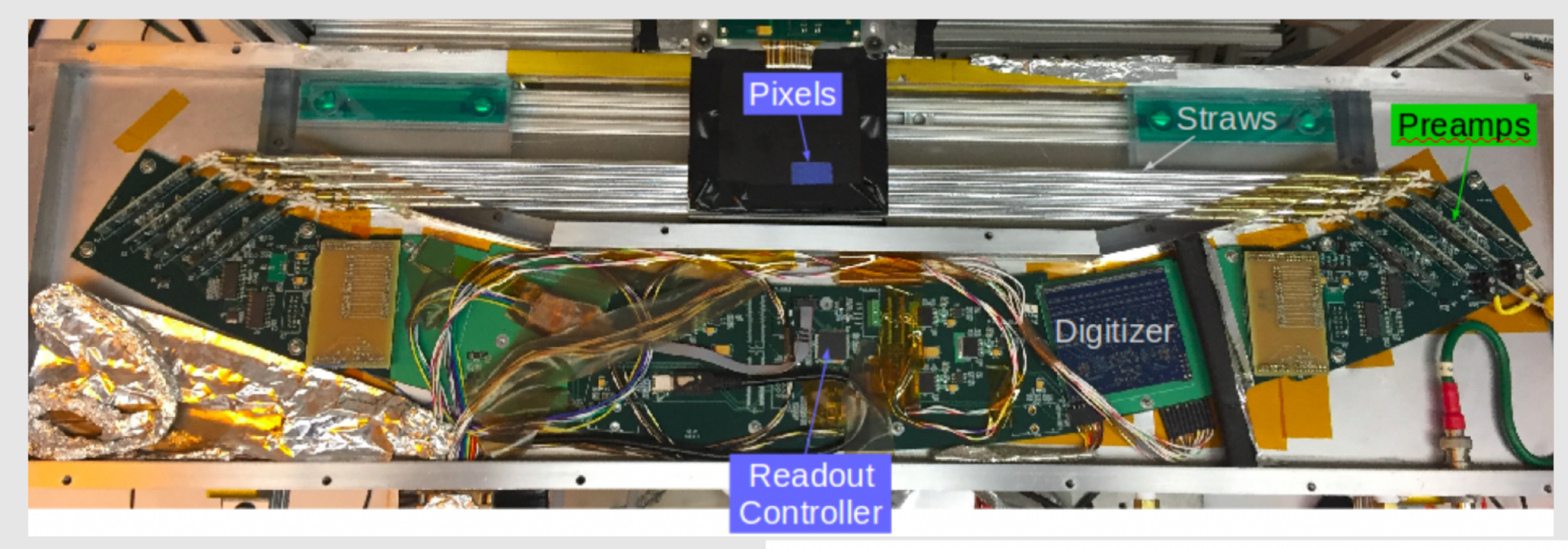

Measured gain, crosstalk, resolution...

- Use Cosmic Rays.

- Use information gained to update MC.

- Measured performance and resolutions.

- Performance met requirements!
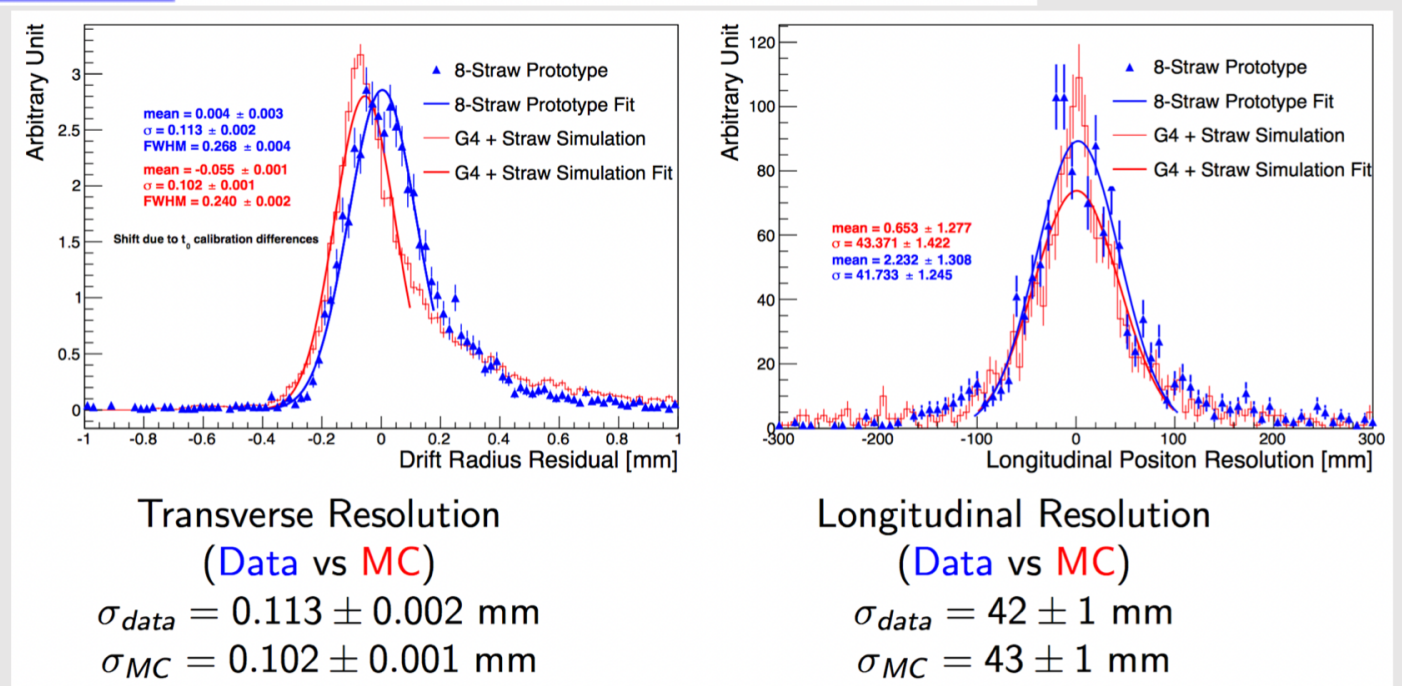
R\&D and Prototyping successfully completed. Crystal and SiPM fabrication has begun. $67 \%$ of crystals, $100 \%$ SiPMs.
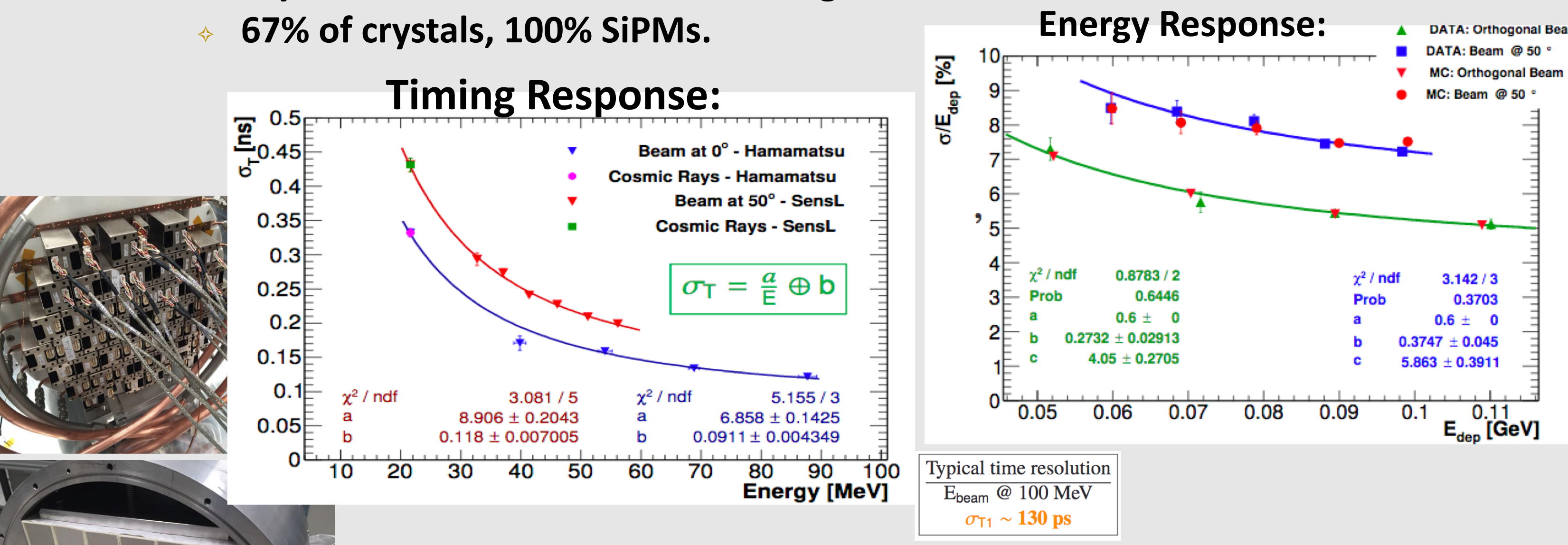

Test beam with $\mathrm{e}^{-}$with $\mathrm{E}=60-120 \mathrm{MeV}$.

Good agreement between MC/Data!

Meets energy and timing performance requirements!

MANCHESTER 1824 


\section{Mu2e \\ What happens if we see a signal?}

V. Cirigliano, S. Davidson, YK, Phys. Lett. B 771 (2017) 242

S. Davidson, YK, A. Saporta, Eur. Phys.

J. C78 (2018) 109

If we do see a signal at Mu2e in Al:

Various operator coefficients add coherently in the amplitude.

Weighted by nucleus-dependent functions.

$\rightarrow$ Requires measurements of $R$ in other target materials!

$5 \%$ measurement on $\mathrm{Al} / \mathrm{Ti}$ needed to see split

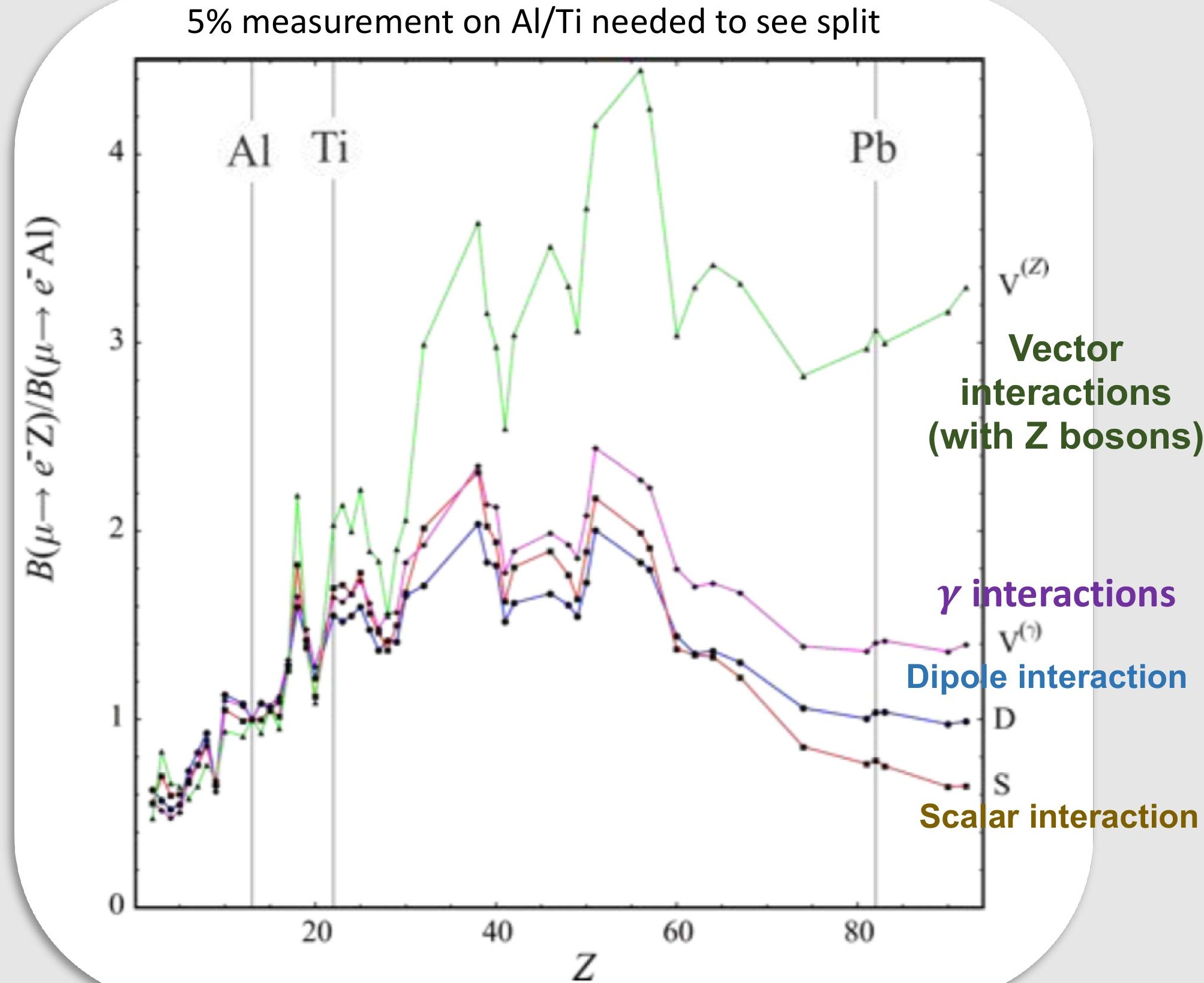




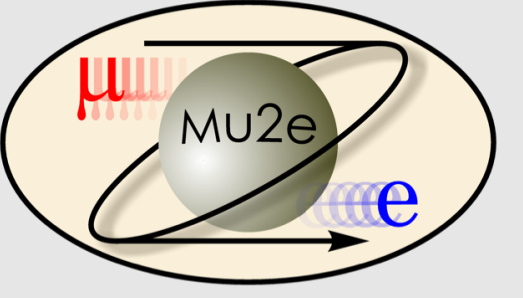

Use $100 \mathrm{~kW}$ of PIP-II protons at $800 \mathrm{MeV}$ to achieve an $\times 10$ improvement in sensitivity.

If there is no signal at Mu2e:

We could extend our sensitivity to find a signal or set new limits.

If Mu2e does see something:

\& Mu2e-II would improve statistical significance, different target materials to narrow down the NP processes.

Mu2e-II could begin taking data around 2030.

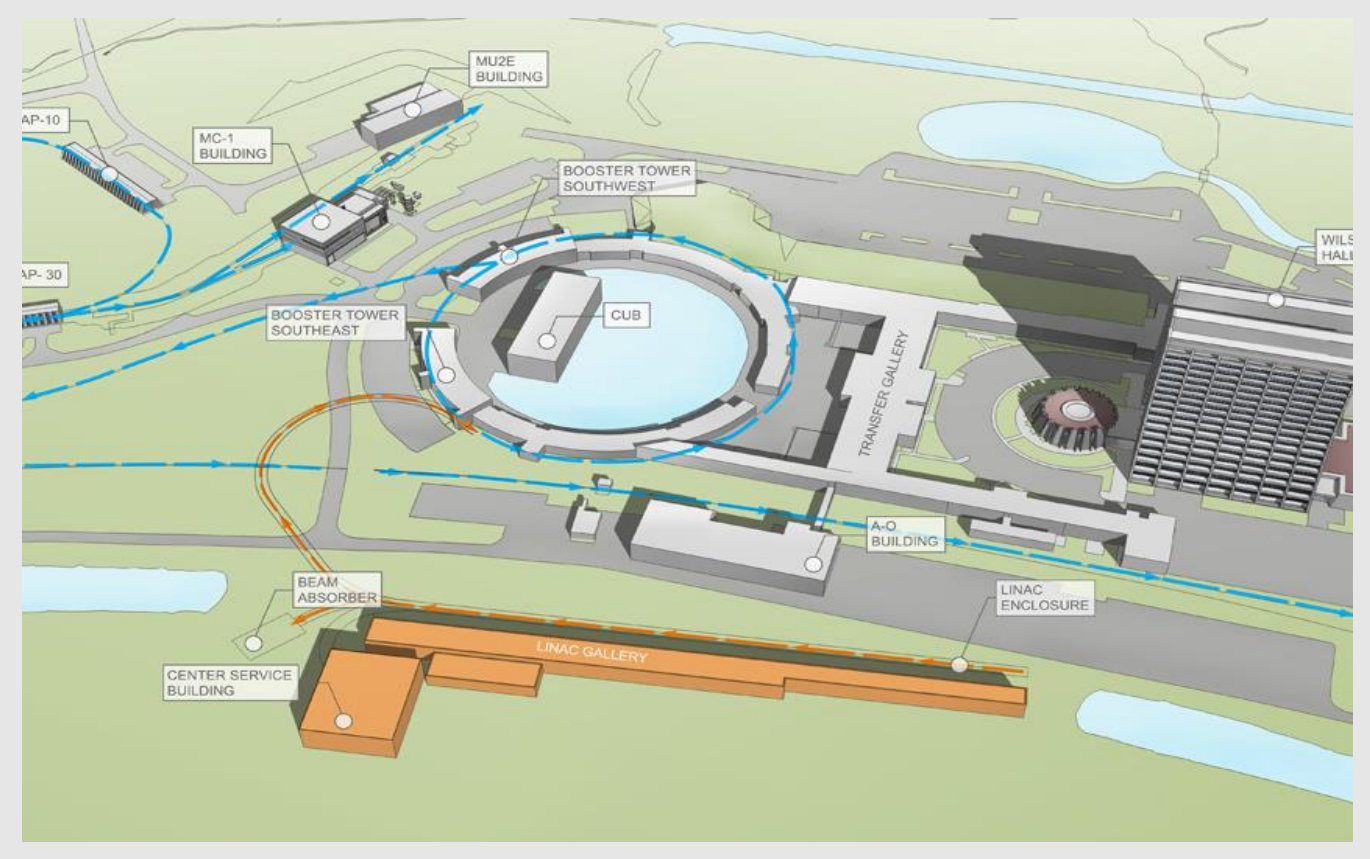


The Mu2e Experiment is a search for CLFV based at Fermilab looking for signal of coherent, neutrinoless conversion of muon to electron in nucleus. Aims:

\& To Improve sensitivity on relative rate to reach $R_{\mu e}<8 \times 10^{-17}$

With SES $3 \times 10^{-17}$

$\diamond$ And $5 \sigma$ discovery at $2 \times 10^{-16}$.

Will constrain New Physics models up to a scale of $10^{4} \mathrm{TeV}$.

Lots of activity currently underway at both FNAL and at our many collaborating institutions.

Commissioning will begin in the next year with physics data taking expected by 2022. 


\section{Thank You For Listening!}

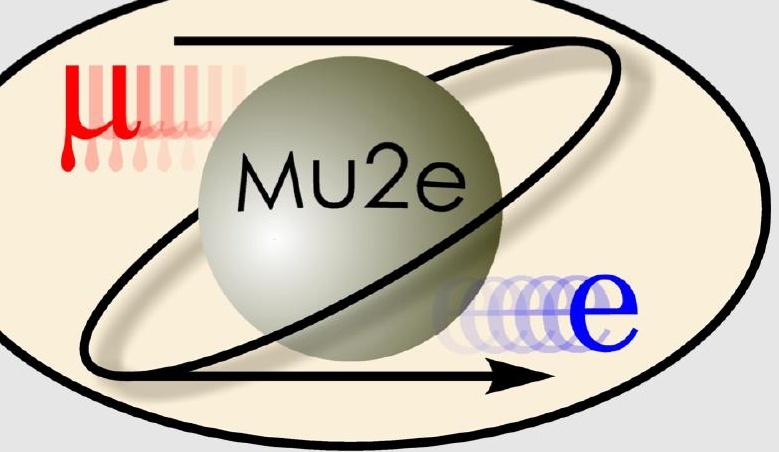

\section{MANCHESTER 1824}

The University of Manchester 


\title{
Back-Up
}

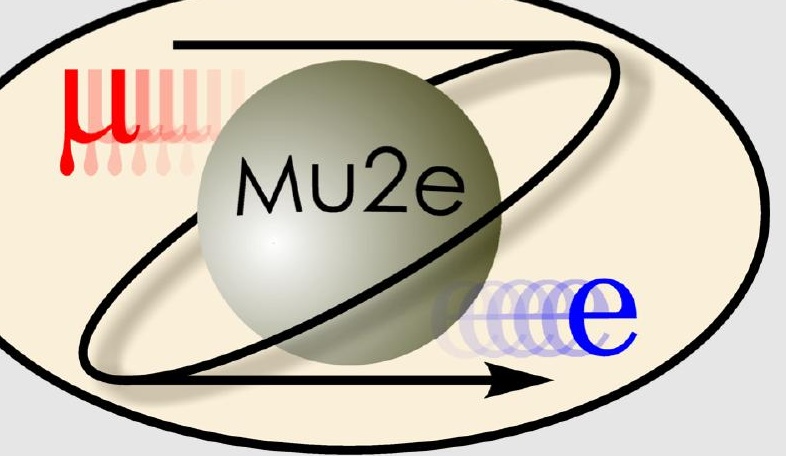

\author{
MANCHESTER \\ 1824
}

The University of Manchester 
\& In Minimal Extension to SM possible in loop diagrams due to neutrino oscillations:

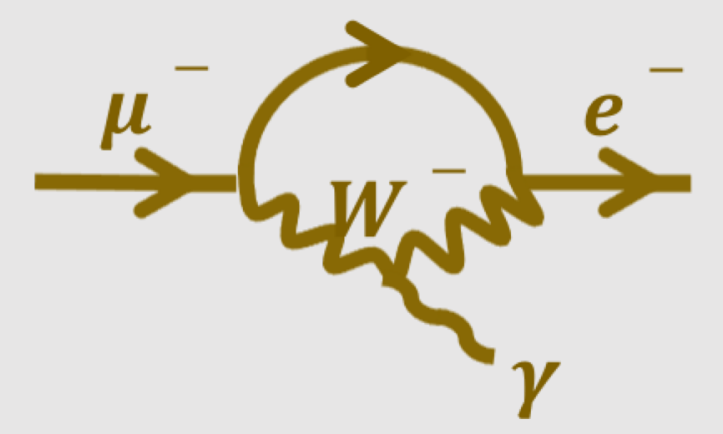

$$
B R(\mu \rightarrow e \gamma)=\frac{3 \alpha}{32 \pi}\left|\sum_{i=2,3} U_{\mu i}^{*} U_{e i} \frac{\Delta m_{1 i}^{2}}{M_{W}^{2}}\right|^{2}<10^{-54}
$$

$$
\mu^{-} N \rightarrow e^{-} N
$$

=> An observation is unambiguous evidence of New

$$
\text { Physics! }
$$

Broad array of New Physics models predict rates observable at next generation CLFV experiments New Physics models predict rates in $10^{-14}-10^{16}$ region.

Mu2e sees 40 conversions at $\mathbf{1 0}^{-15}$. 


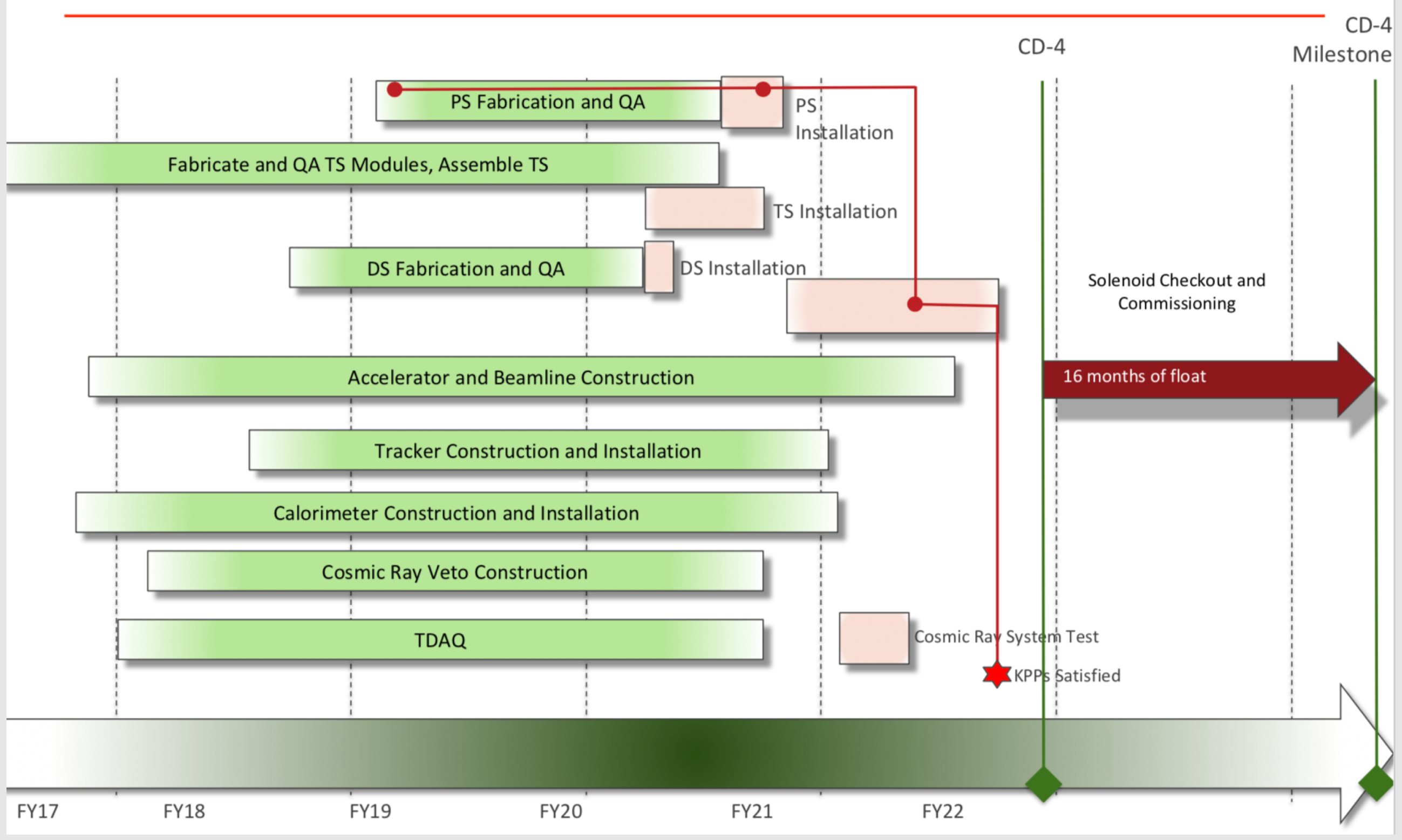




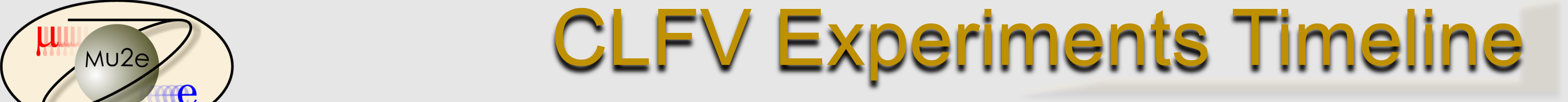

Searches for Charged-Lepton Flavor Violation in Experiments using Intense Muon Beams

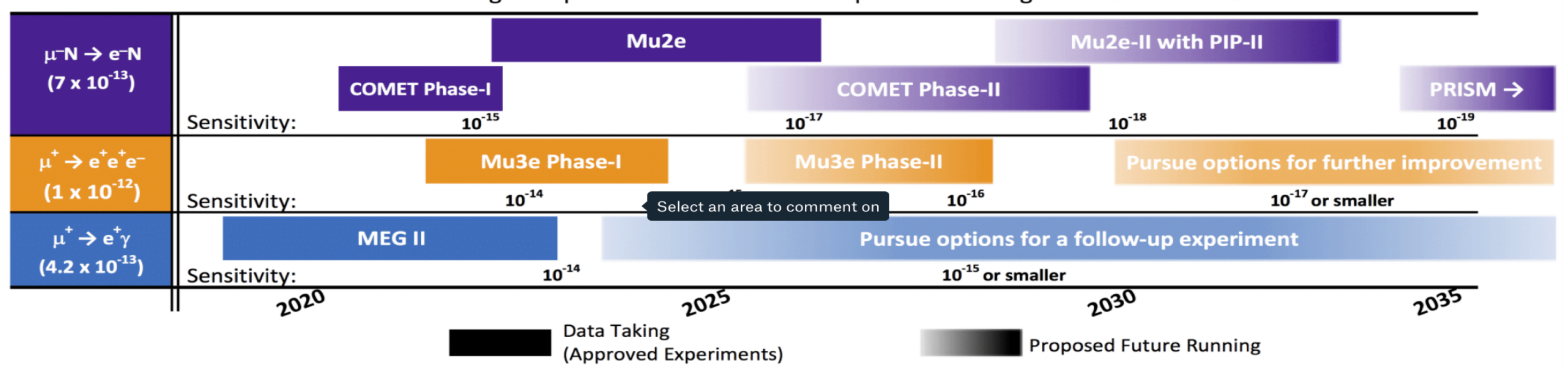

Figure 1: Planned data taking schedules for current experiments that search for charged-lepton flavor violating $\mu \rightarrow e$ transitions. Also shown are possible schedules for future proposed upgrades to these experiments. The current best limits for each process are shown on the left in parentheses, while expected future sensitivities are indicated by order of magnitude along the bottom of each row.

*Y. Kuno

\section{MANCHESTER 1824}




\section{1

Mu2e uses $8 \mathrm{~kW}$ of $8 \mathrm{GeV}$ protons from the Booster.

2 batches of $4 \times 1012$ Protons, transported from Booster via MI- 8 beamline to the Recycler Ring Circulate and re-bunched by a $2.5 \mathrm{MHz}$ RF system.

Reformatted bunches are kicked into the P1 line and transported to the Delivery Ring They are slow extracted to the Mu2e detector through a new external beamline

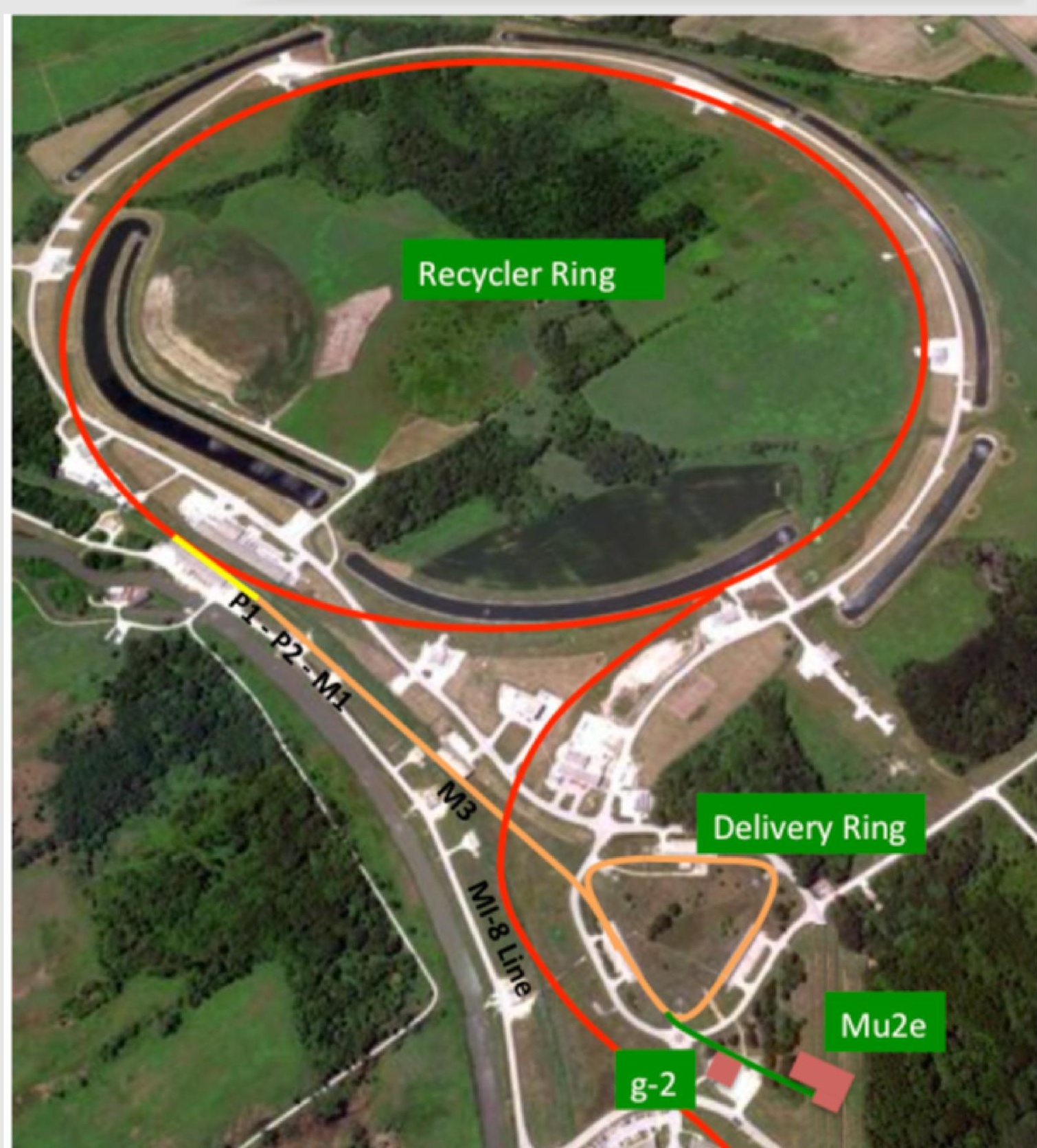




\section{Where will our muons come from?}
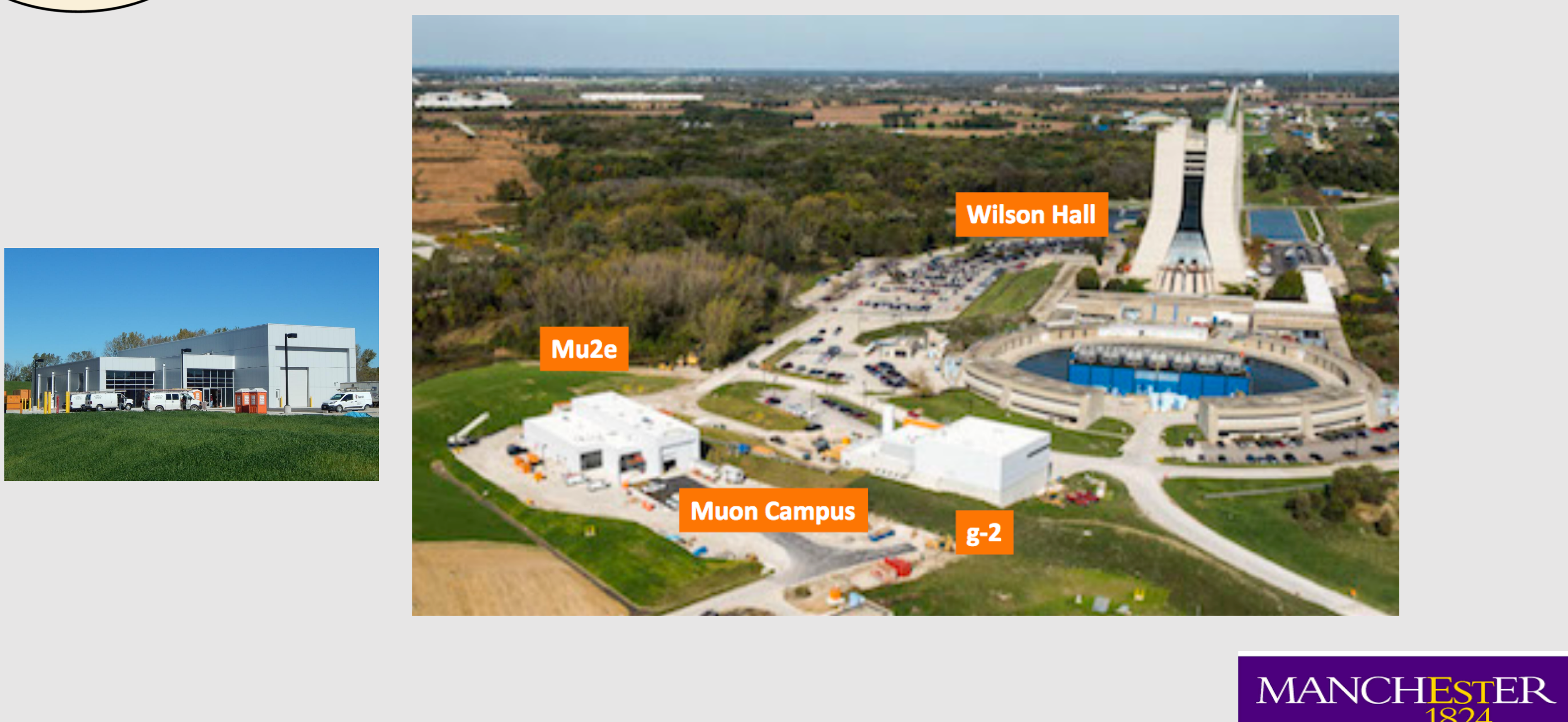

The University of Manchester 


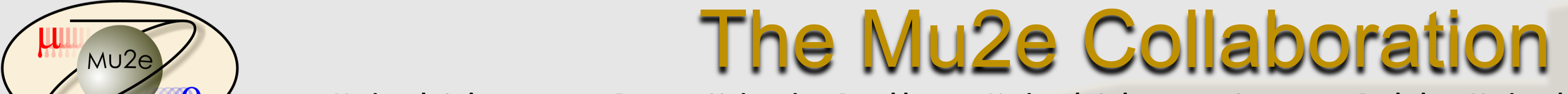

Argonne National Laboratory - Boston University Brookhaven National Laboratory Lawrence Berkeley National Laboratory and University of California, Berkeley • University of California, Davis • University of California, Irvine • California Institute of Technology • City University of New York • Joint Institute for Nuclear Research, Dubna • Duke University • Fermi National Accelerator Laboratory • Laboratori Nazionali di Frascati • INFN Genova • HelmholtzZentrum Dresden- Rossendorf • University of Houston • Institute for High Energy Physics, Protvino • Kansas State University • INFN Lecce and Università del Salento • Lewis University • University of Liverpool • University College London • University of Louisville • University of Manchester • Laboratori Nazionali di Frascati and Università Marconi Roma • University of Minnesota • Institute for Nuclear Research, Moscow • Muons Inc. • Northern Illinois University • Northwestern University • Novosibirsk State University/Budker Institute of Nuclear Physics • INFN Pisa • Purdue University • University of South Alabama • Sun Yat Sen University • University of Virginia • University of Washington •

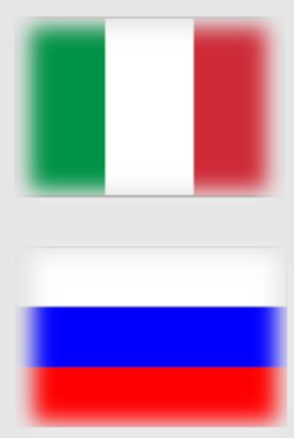
Yale University

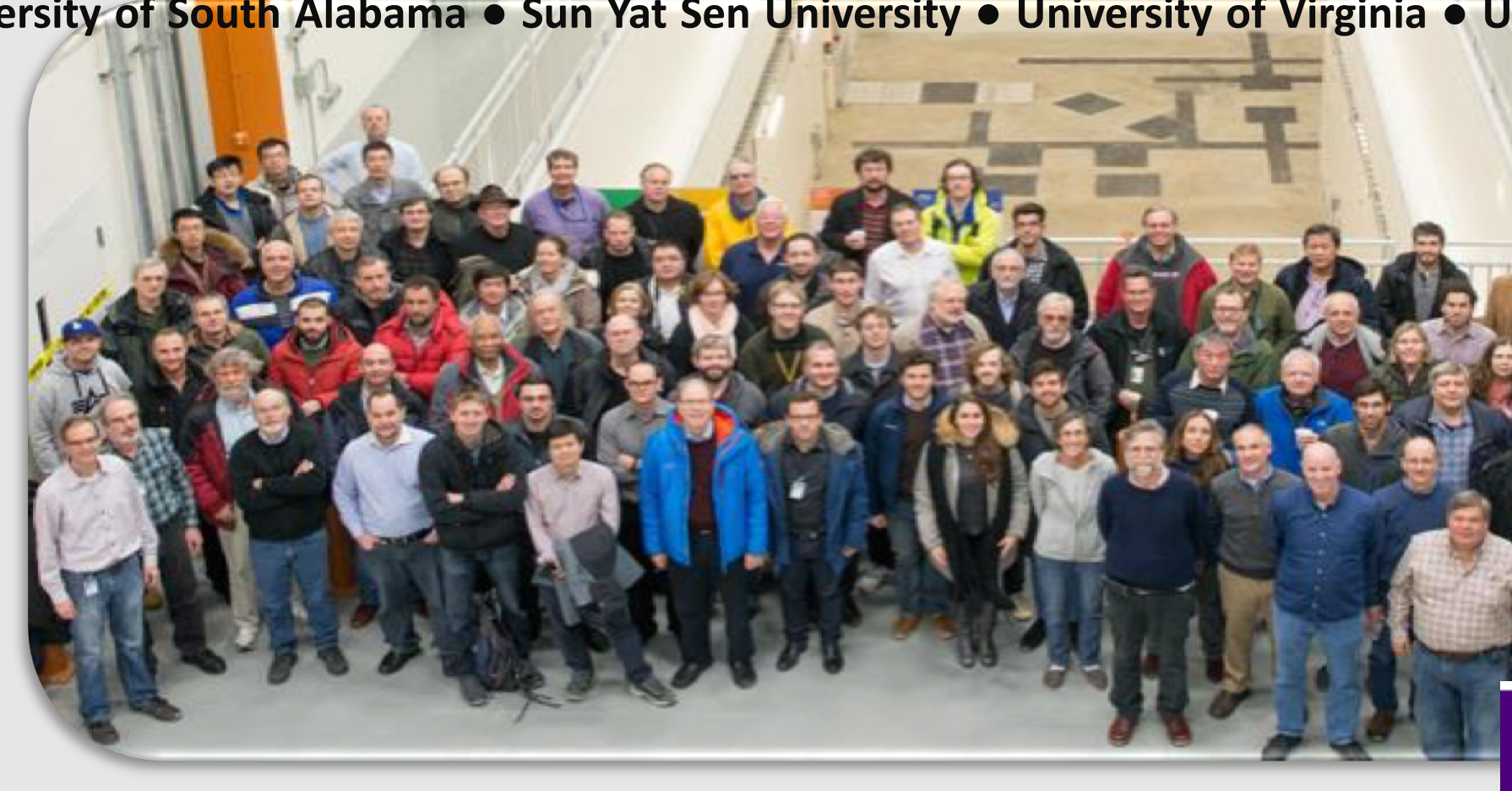




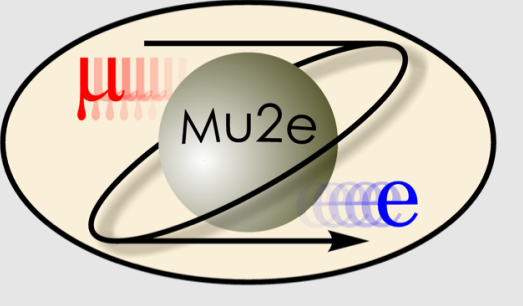

$\Leftrightarrow$ Must have out-of-time : in-time proton ratio $<10^{-10}$

2 steps:

1. The technique for generating the required bunch structure in the Recycler Ring $y$ leads to a high level of extinction. Fast "kicker" which transfers the proton beam from the Recycler to the Delivery Ring preserves extinction. Extinction of $10^{-5}$ is expected as the proton beam is extracted and delivered.

2. The beam line from the Delivery Ring to the production target has a set of $A C$ oscillating dipoles that sweep out-of-time protons into a system of collimators. This should achieve an additional extinction of $10^{-7}$ or better. 

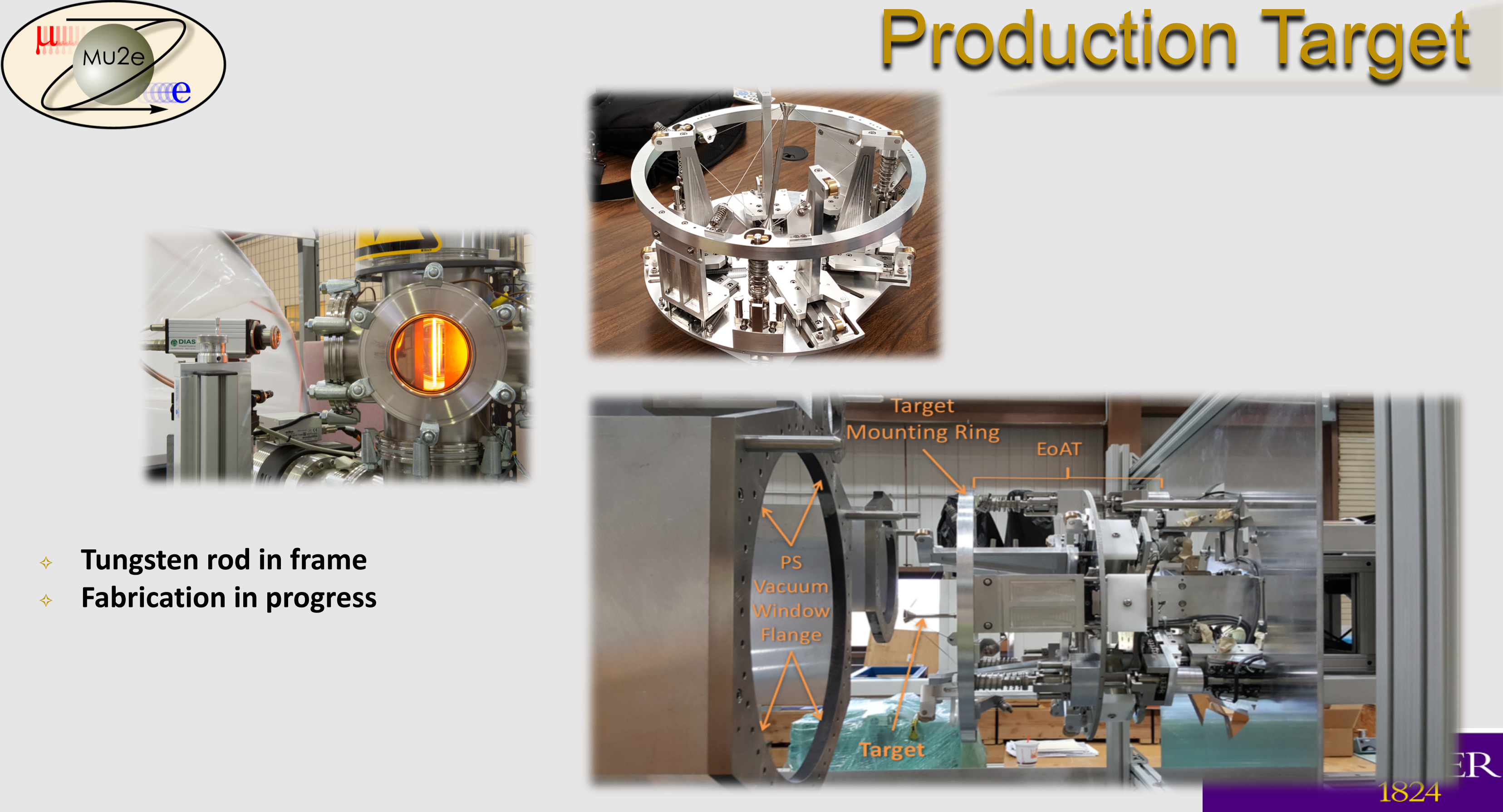

Tungsten rod in frame Fabrication in progress 
Built at ASG \& Shipped to Fermilab Successful R\&D and prototype campaign completed. Vendor, ASG, has delivered $25 \%$ of the 27 TS coil modules.

$100 \%$ of the 52 TS coils wound at cold mass vendor. Good progress made with the fabrication of the coils' housing shells

FNAL solenoid test facility operational.

The first of the coil modules has been installed around the magnet bore at assembly site.

$\diamond$ Fabrication of the outer TS cryostat is underway.
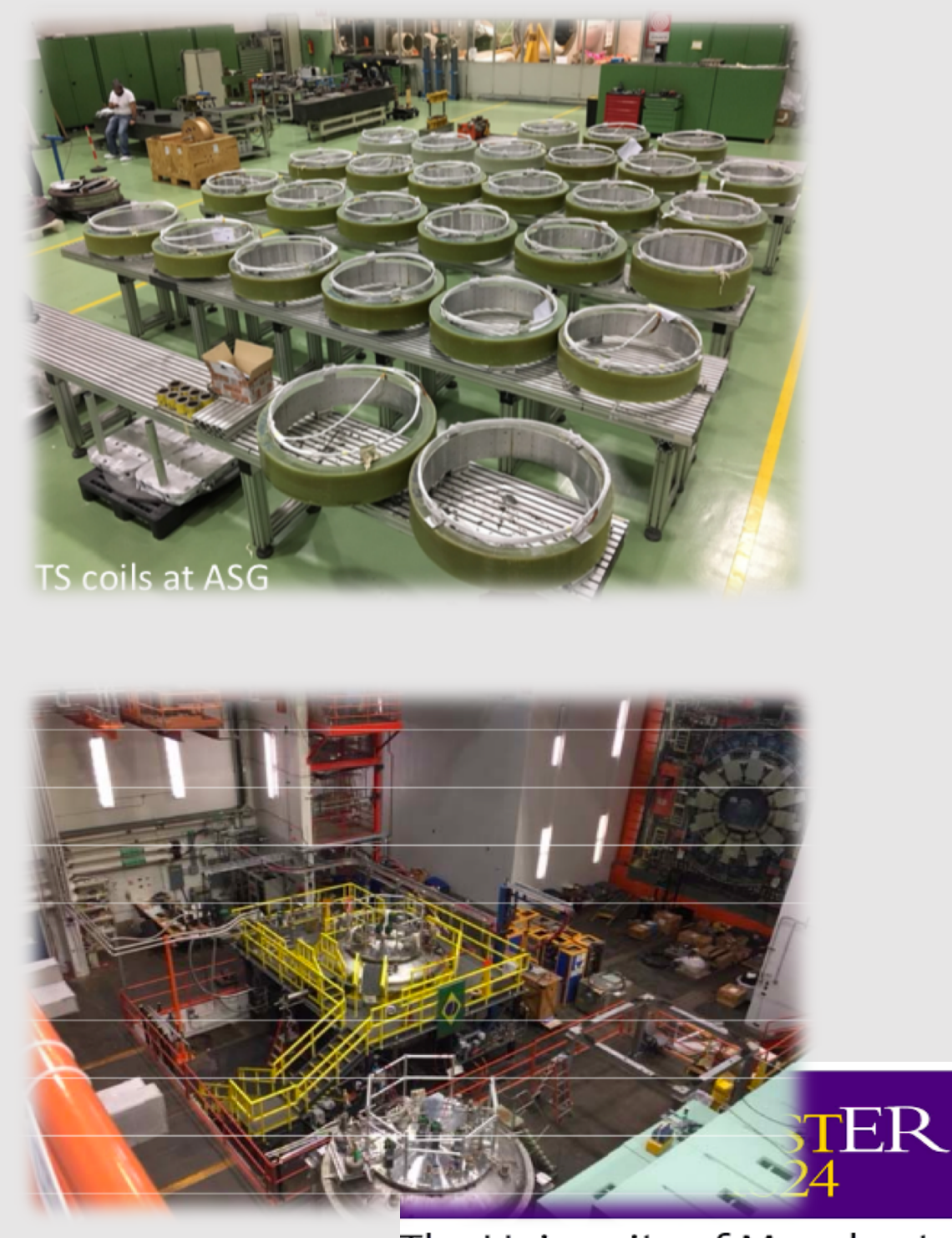
Recent Solenoid Progress at FNAL

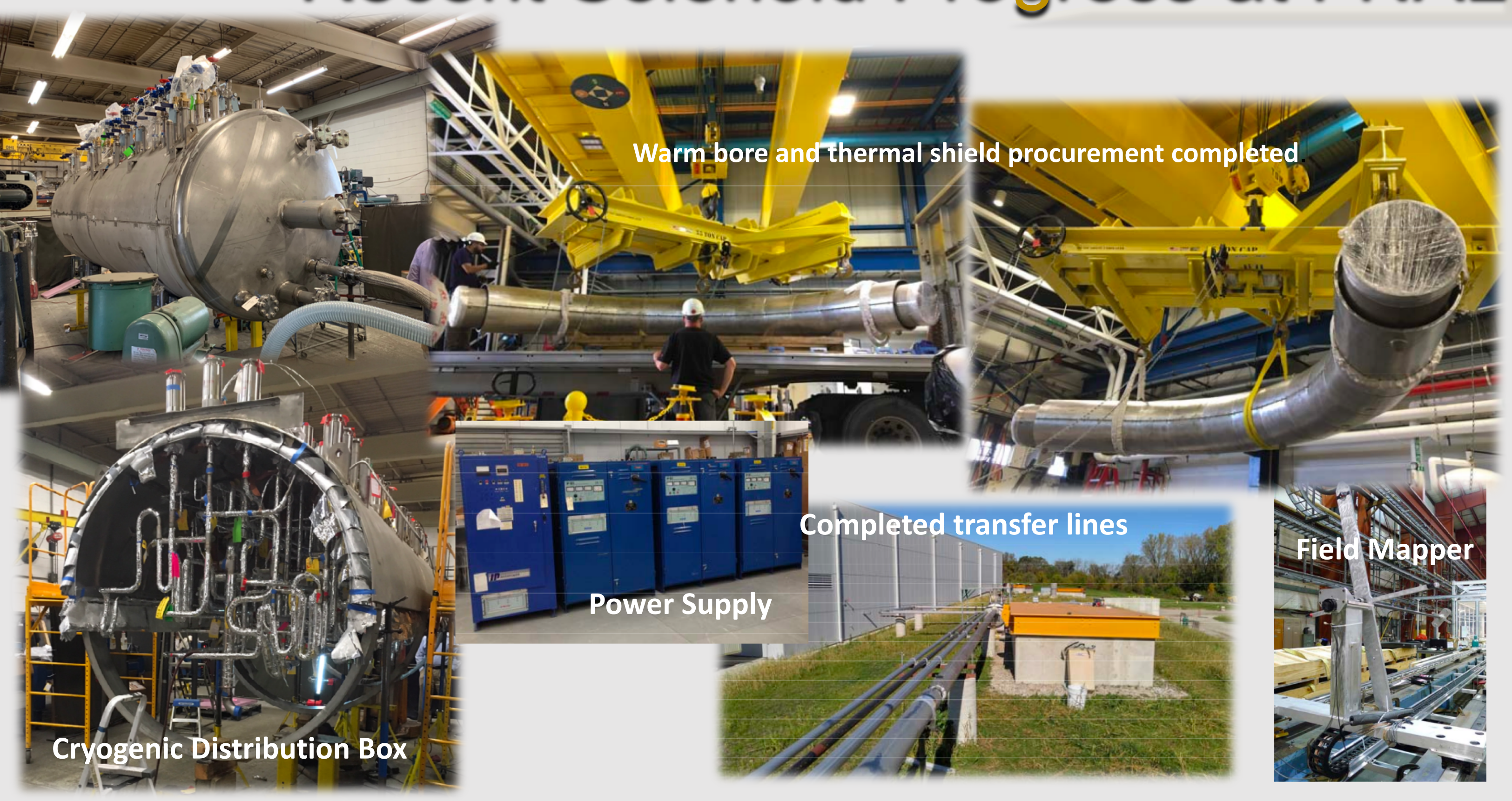




\section{$=$

Need an accurate measure of total number of stopped muons in the target (within $10 \%)$. Placed far downstream of DS ( $34 \mathrm{~m}$ from target).

STM uses HPGe and $\mathrm{LaBr}_{3}$ detectors to measure X/gamma-rays produced by stopped muons in Al target:

Prompt X-ray emitted from muonic atoms at 347keV;

Semi-prompt gamma ray at $1.809 \mathrm{MeV}$;

Delayed gamma ray at $844 \mathrm{keV}$.
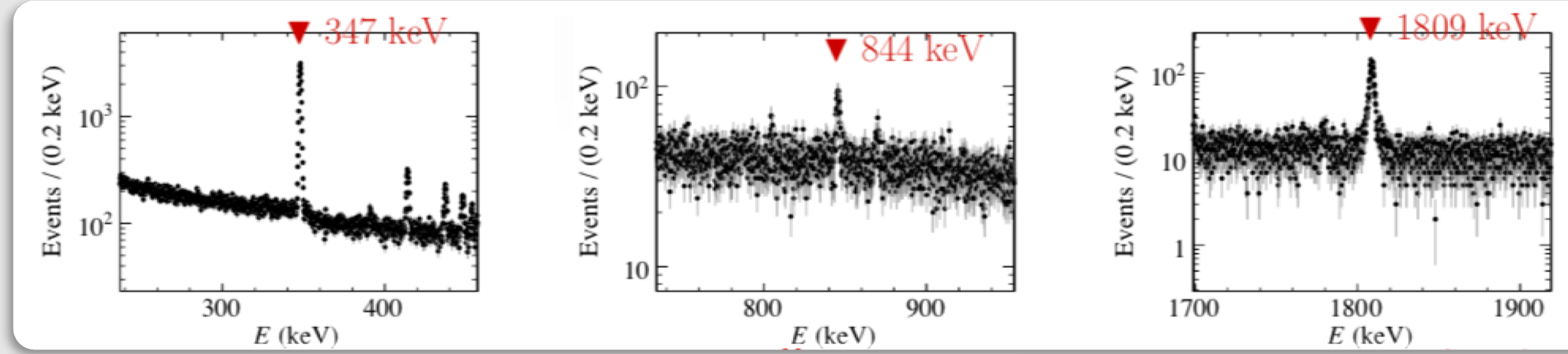

MANCHESTER 1824 


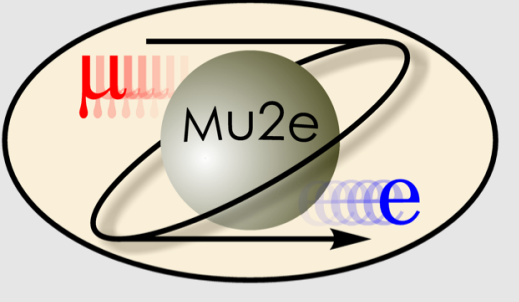

The DAQ: Purpose

The DAQ system must provide readout and control for all detector subsystems.

Trigger processing is handled entirely in software.

This allows use of commercial computing hardware.

Filtering can be designed in offline environment and can then be run in online trigger environment.

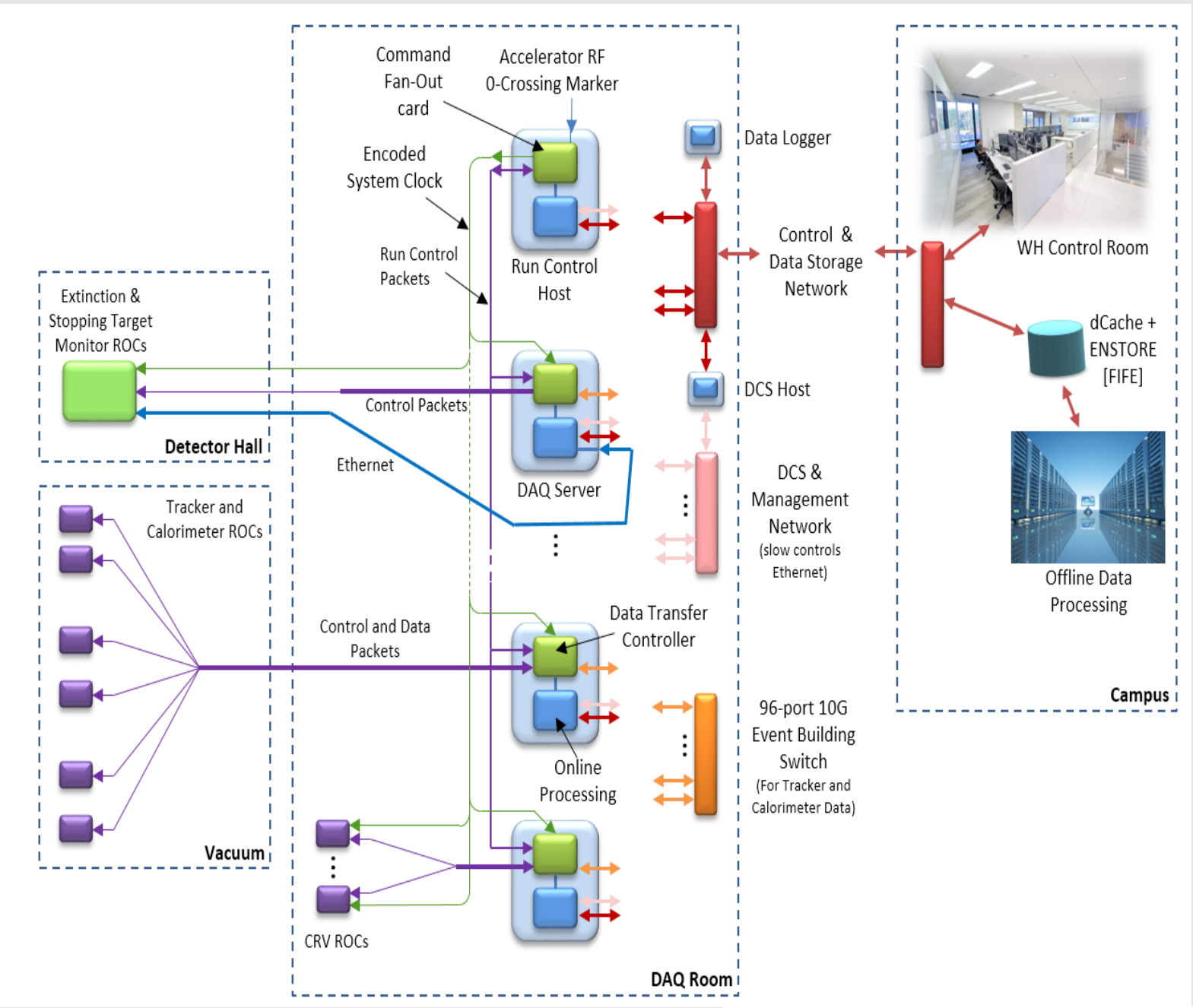

MANCHESTER 1824 


\section{Relative rates important in determining new} physics:

\begin{tabular}{|c|c|c|c|c|}
\hline Model & $\mu \rightarrow$ eee & $\mu N \rightarrow e N$ & $\frac{\mathrm{BR}(\mu \rightarrow e e e)}{\mathrm{BR}(\mu \rightarrow e \gamma)}$ & $\frac{\mathrm{CR}(\mu N \rightarrow e N)}{\mathrm{BR}(\mu \rightarrow e \gamma)}$ \\
\hline MSSM & Loop & Loop & $\approx 6 \times 10^{-3}$ & $10^{-3}-10^{-2}$ \\
\hline Type-I seesaw & Loop* & Loop* & $3 \times 10^{-3}-0.3$ & $0.1-10$ \\
\hline Type-II seesaw & Tree & Loop & $(0.1-3) \times 10^{3}$ & $\mathcal{O}\left(10^{-2}\right)$ \\
\hline Type-III seesaw & Tree & Tree & $\approx 10^{3}$ & $\mathcal{O}\left(10^{3}\right)$ \\
\hline LFV Higgs & Loop $^{\dagger}$ & Loop $^{* \dagger}$ & $\approx 10^{-2}$ & $\mathcal{O}(0.1)$ \\
\hline Composite Higgs & Loop* & Loop* & $0.05-0.5$ & $2-20$ \\
\hline
\end{tabular}

from L. Calibbi and G. Signorelli, Riv. Nuovo Cimento, 41 (2018) 71

\section{MANCHESTER 1824}




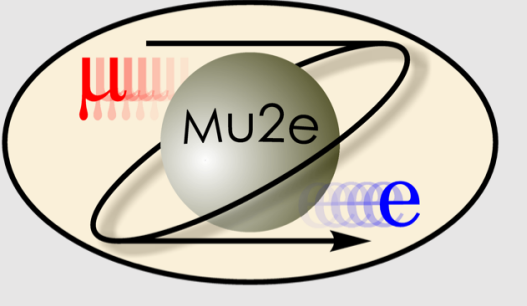

\section{Constraining NP with CLFV SUSY SO(10) GUT:}

Interesting Overviews:

Calibbi L, Signorelli G. Charged lepton flavour violation: anexperimental and theoretical introduction. Riv Nuovo Cim.(2018) 41:71.

Gouvêa A, Vogel P. Lepton flavor and number conservation, and physics beyond the standard model.arXiv:1303.4097 (2013)

Marciano WJ, Mori T, Roney JM. Charged lepton flavour violation experiments. Annu. Rev. Nucl. Part. Sci. (2008)

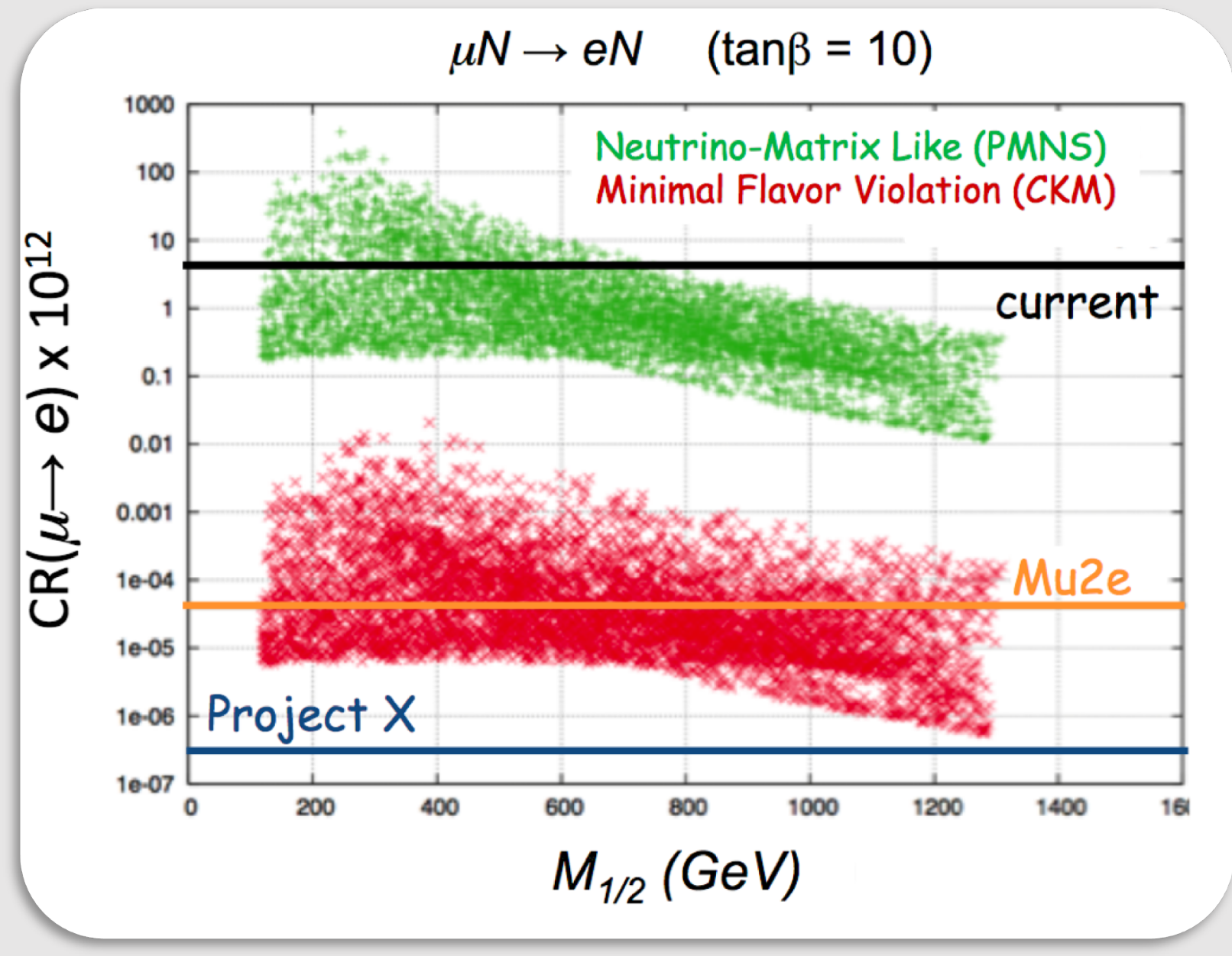

MANCHESTER 


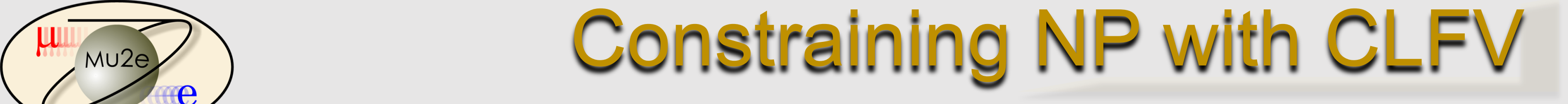

\section{Scalar Leptoquarks}

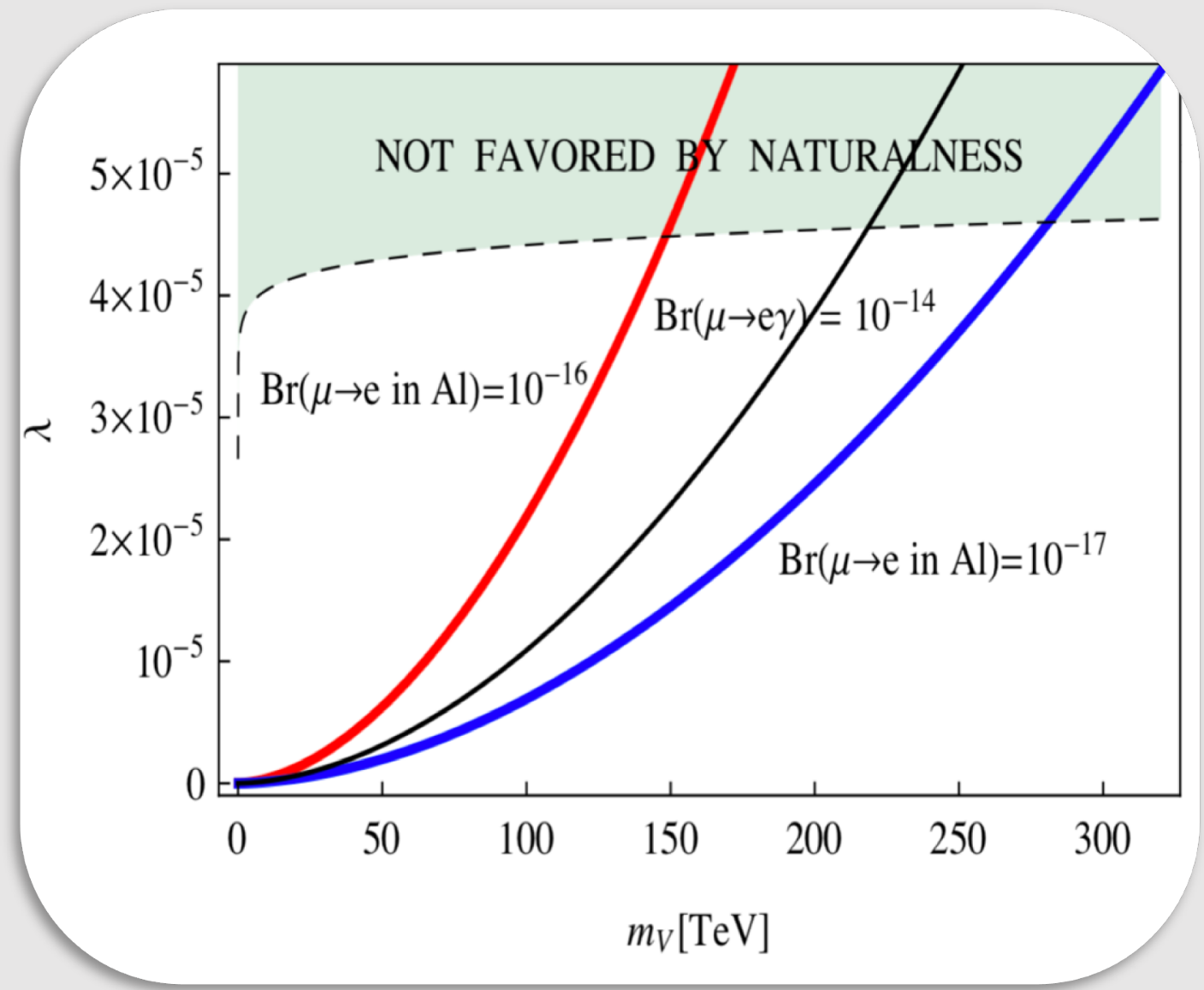

Figure shows the reach in the new coupling $\lambda$ for a range of scalar leptoquark masses for the $\mu \rightarrow$ e conversion rate for two values of the $\operatorname{Br}(\mu \rightarrow$ e conversion in Al) relevant for the Mu2e experiment. 